\title{
Review
}

Jinghan He, Hong Chen, Jin Hu, Jingan Zhou, Yingmu Zhang, Andre Kovach, Constantine Sideris, Mark C. Harrison, Yuji Zhao and Andrea M. Armani*

\section{Nonlinear nanophotonic devices in the ultraviolet to visible wavelength range}

https://doi.org/10.1515/nanoph-2020-0231

Received April 7, 2020; accepted June 12, 2020; published online July 4, 2020

\begin{abstract}
Although the first lasers invented operated in the visible, the first on-chip devices were optimized for near-infrared (IR) performance driven by demand in telecommunications. However, as the applications of integrated photonics has broadened, the wavelength demand has as well, and we are now returning to the visible (Vis) and pushing into the ultraviolet (UV). This shift has required innovations in device design and in materials as well as leveraging nonlinear behavior to reach these wavelengths. This review discusses the key nonlinear phenomena that can be used as well as presents several emerging material systems and devices that have reached the UV-Vis wavelength range.
\end{abstract}

Keywords: inverse design; nanophotonics; nonlinear optics; optical materials; organic materials.

\footnotetext{
*Corresponding author: Andrea M. Armani, Dept. of Chemistry, University of Southern California, Los Angeles, CA, 90089, USA; Ming Hsieh Dept. of Electrical and Computer Engineering, University of Southern California, Los Angeles, CA, 90089, USA; and Mork Family Dept. of Chemical Engineering and Materials Science, University of Southern California, Los Angeles, CA, 90089, USA, E-mail: armani@usc.edu. https://orcid.org/0000-0001-9890-5104 Jinghan He: Dept. of Chemistry, University of Southern California, Los Angeles, CA, 90089, USA. https://orcid.org/0000-0001-7713-1226

Hong Chen, Jingan Zhou and Yuji Zhao: School of Electrical, Computer, and Energy Engineering, Arizona State University, Tempe, AZ, 85287, USA

Jin Hu and Constantine Sideris: Ming Hsieh Dept. of Electrical and Computer Engineering, University of Southern California, Los Angeles, CA, 90089, USA

Yingmu Zhang and Andre Kovach: Mork Family Dept. of Chemical Engineering and Materials Science, University of Southern California, Los Angeles, CA, 90089, USA

Mark C. Harrison: Fowler School of Engineering, Chapman University, Orange, CA, 92866, USA
}

Әopen Access. @ 2020 Jinghan He et al., published by De Gruyter. (cc) BY License.

\section{Introduction}

The past several decades has witnessed the convergence of novel nonlinear materials with nanofabrication methods, enabling a plethora of new nonlinear optical (NLO) devices [1-4]. Originally, the focus was on developing devices operating in the telecommunications wavelength band to improve communications. One example of an initial success is on-chip modulators and add-drop filters for switching and isolating of optical wavelengths. While initial devices were fabricated from crystalline materials [5-7], the highest performing devices were made from organic polymers [8-16]. As nanofabrication processes improved, higher performance integrated devices were developed, such as high quality factor optical resonant cavities, and higher order nonlinear behaviors became accessible. This technology enabled on-chip frequency combs [2, 17, 18], stokes and anti-stokes lasers [19-21], and super continuum sources [22].

While these devices can be used in many fields, one clear application of these devices is in quantum optics. While many quantum phenomena can be investigated using near-infrared (IR) lasers, atomic clocks based on $\mathrm{Rb}$ and Ce transition lines require visible lasers as excitation sources. Initial work developing proof of concept systems relied on large optical lasers. More recently, the focus shifted to "clocks on a chip" [23-26]. Because the transition lines are in the visible, the development of an ultra-narrow linewidth and stable visible laser source at a complementary wavelength was a key stepping stone. Similarly, over the past few years, a plethora of novel quantum emitters have been discovered [27]. However, the majority are excited in the visible. In order to realize integrated devices based on these new materials, it is necessary to have an integrated source with sufficient power.

Visible sources also play a key role in biotechnology, namely, the miniaturization of diagnostics and imaging systems. Tissue and biosamples absorb strongly in the near-IR wavelength range. This absorbance will degrade the performance of many diagnostic techniques, and it can result in scattering and signal degradation in imaging 
[28-30]. In addition, many imaging methods require fluorescent probes. The majority of light emitting bio-labels are excited in the ultraviolet (UV) to visible wavelength range $(\sim 300-\sim 700 \mathrm{~nm})$ [31-33]. Therefore, to support this rapidly emerging field, there is a growing effort to develop complementary integrated sources.

This review will introduce the key theoretical mechanisms that underpin nonlinear interactions in integrated photonic devices. These act as design rules for both the devices discussed here as well as devices in general. Then, a discussion of several new crystalline and organic materials and devices being actively used to achieve ultraviolet (UV)visible (Vis) emission with be reviewed. Lastly, a discussion of possible new research directions will be presented.

\section{Basics of nonlinear optics}

\subsection{Background}

Maxwell's equations form the basis for describing electric and magnetic fields at a macroscopic level. Combining them, one can obtain the wave equation which is the foundation for electromagnetic radiation, also known as light. Two of Maxwell's equations for electric displacement (D) and magnetic field $(\mathrm{H})$ are given below:

$$
\begin{gathered}
\nabla \cdot D=\rho_{f} \\
\nabla \tilde{n} H=j_{f}+\frac{\partial D}{\partial t}
\end{gathered}
$$

where $\rho_{f}$ is the free charge density, and $j_{f}$ is the free current density. Furthermore, $\mathbf{D}$ and $\mathbf{H}$ can be obtained through the constitutive relationships. We will focus on $\mathbf{D}$, because most materials (and the materials discussed herein) are nonmagnetic, so $\mathbf{H}$ is directly related to $\mathbf{B}$ via $\mu_{0}$. The electric displacement, D, is related to the electric field, E, via the permittivity $\left(\varepsilon_{0}\right)$ and the polarization density $(\boldsymbol{P})$ :

$$
D=\epsilon_{0} E+P
$$

Furthermore, the polarization can be represented by a sum of its linear and nonlinear parts:

$$
P=P^{(L)}+P^{(N L)}
$$

Assuming a plane-wave propagating in the $z$ direction with amplitude $A$, angular frequency $\omega$, and propagation vector $k$, we use the wave equation to relate the field amplitude to nonlinear polarization. Applying the slowlyvarying approximation (the field varies slowly with propagation distance), which applies in most nonlinear materials, and assuming negligible loss we arrive at:

$$
\frac{d A(\omega)}{d z}=j \frac{\omega}{2 n \epsilon_{0} c} P^{(N L)}(\omega) e^{-j k z}
$$

In other words, the amplitude of the field as a function of frequency will vary depending on the nonlinear polarization density, which is medium dependent. The particular form of the nonlinear component of polarization will depend on the nonlinear process generated in the material, but in general the polarization density can be expanded in a power series. The first term represents the linear part, and all subsequent terms represent the nonlinear part:

$$
P=\epsilon_{0} \chi^{(1)} E+\epsilon_{0} \chi^{(2)} E^{2}+\epsilon_{0} \chi^{(3)} E^{3}+\ldots
$$

The $\chi$ terms represent different orders of nonlinear susceptibility, and each is a tensor with terms to mix the $x$, $y$, and $z$ components of the electric field. Additionally, Eq. (6) is only valid in the frequency domain or for ultrafast nonlinearities in non-dispersive materials. In other instances in the time domain, overlap integrals with the response time are required, but for our simple analysis, Eq. (6) will suffice. We will explore specific components of nonlinear polarization in the following sections and discus how they can be leveraged to generate frequencies in the UV-Vis range.

\section{$2.2 \chi^{(2)}$ effects: second harmonic generation and three wave mixing}

In this section, we focus on the second-order terms, which correspond to the $\chi^{(2)}$ expansion term, and are thus often referred to as $\chi^{(2)}$ effects. The $\chi^{(2)}$ coefficient is often represented mathematically with the $d$ coefficient $\left(d=\frac{1}{2} \chi^{(2)}\right)$. Since both of these quantities tensors, they include terms to mix the $x, y$, and $z$ components. The $\chi^{(2)}$ effects collectively include several 3-wave mixing effects: second-harmonic generation (SHG or frequency doubling), differencefrequency generation, and sum-frequency generation to name a few. For simplicity, we treat these effects together. For $\chi^{(2)}$ effects, we first consider three frequencies of light traveling through a crystal (Figure 1a) such that $\omega_{3}=\omega_{1}+\omega_{2}$. Now we examine the coupled wave equations that result from nonlinear polarization density being a tensor and consider only the frequencies that satisfy the enforced constraint $\omega_{3}=\omega_{1}+\omega_{2}$. The resulting coupled wave equations are:

$$
\frac{d}{d z} E_{1}(z)=-j \omega_{1} \sqrt{\frac{\mu_{0}}{\epsilon_{1}}} d E_{3} E_{2}^{*} e^{-j\left(k_{3}-k_{2}-k_{1}\right) z}
$$



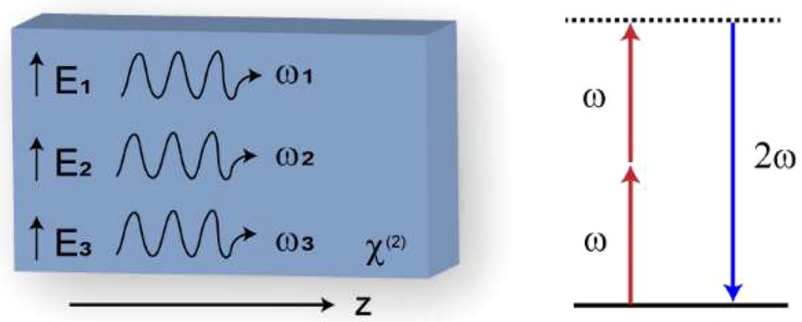

(a)

(b)

Figure 1: (a) Three waves with frequencies $\omega_{1}, \omega_{2}$, and $\omega_{3}$ mix in a nonlinear optical crystal exhibiting second-order $\left(x^{(2)}\right)$ effects, propagating in the $z$-direction. (b) For second-harmonic generation (SHG), two photons at the pump frequency are destroyed while interacting with the material to produce a single photon at double the pump frequency (half the wavelength).

$$
\begin{aligned}
& \frac{d}{d z} E_{2}(z)=-j \omega_{2} \sqrt{\frac{\mu_{0}}{\epsilon_{2}}} d E_{3} E_{1}^{*} e^{-j\left(k_{3}-k_{2}-k_{1}\right) z} \\
& \frac{d}{d z} E_{3}(z)=-j \omega_{3} \sqrt{\frac{\mu_{0}}{\epsilon_{3}}} d E_{2} E_{1} e^{-j\left(k_{3}-k_{2}-k_{1}\right) z}
\end{aligned}
$$

For propagation through thin materials, we assume that the pump waves $\left(\omega_{1}\right.$ and $\left.\omega_{2}\right)$ are not depleted, therefore $\frac{d}{d z} E_{1}(z)=\frac{d}{d z} E_{2}(z)=0$. We integrate (Eq. (9)) over $\mathrm{z}$ from 0 to $L$ (with $L$ being the length propagated through the nonlinear material) and assume $E_{3}(O)=O\left(\omega_{3}\right.$ is the frequency being generated). Substituting the $\mathbf{E}$ fields for intensity $\left(I=\frac{n}{2 \sqrt{\frac{\mu_{0}}{\varepsilon_{0}}}} E E^{*}\right)$ we can write the optical intensity as:

$$
I_{3}(L)=2\left(\frac{\mu_{0}}{\epsilon_{0}}\right)^{\frac{3}{2}} \frac{\left(\omega_{3} d L\right)^{2}}{n_{1} n_{2} n_{3}} I_{1} I_{2}\left[\frac{\sin \left(\frac{\Delta k L}{2}\right)}{\frac{\Delta k L}{2}}\right]^{2}
$$

Where

$$
\Delta k=k_{3}-k_{2}-k_{1}=\left[\omega_{3} n_{3}-\omega_{2} n_{2}-\omega_{1} n_{1}\right] / c
$$

$n$ is the frequency-dependent index of refraction, and $c$ is the speed of light in vacuum. It is important to notice that the intensity will vary with a regular beat length due to the presence of the sine function. This regular sinusoidal variation of intensity (or power) over distance is sometimes referred to as power cycling, and it limits nonlinear conversion efficiencies. We will discuss how to address that with phase matching in a subsequent section, but for now, let us consider a special case for the chosen frequencies. For frequency doubling (SHG), we will let $\omega_{1}=\omega_{2}=\omega$ and $\omega_{3}=2 \omega$ and reconsider Eq. (10). In the process of SHG, two photons at frequency $\omega$ are destroyed to create a single photon at frequency $2 \omega$ (Figure $1 b)$.

$$
I_{2 \omega}(L)=2\left(\frac{\mu_{0}}{\epsilon_{0}}\right)^{\frac{3}{2}} \frac{(\omega d L)^{2}}{n_{\omega}^{2} n_{\omega}} I_{\omega}^{2} \operatorname{sinc}^{2}\left[\frac{\Delta k L}{2}\right]
$$

In order to let the intensity of $\omega_{3}$ grow beyond what is limited by the sinc function (that is, beyond the maximum allowed by the periodic variation over distance), we must get $\Delta k=0$, and the way to achieve that is to match the phase of the mixed waves. This is conventionally done by taking advantage of the anisotropic nature of most nonlinear optical materials, but in nanophotonics in particular, it can also be done via modal phase matching or dispersion engineering. The anisotropic nature means that the permittivity, refractive index, and therefore propagation vector $k$ are tensors and will be a function of the polarization of the $\mathbf{E}$ field. The mechanics of phase matching are not discussed here, but we examine it briefly in Section 2.4. With proper alignment of the optical fields with the nonlinear medium and phase matching, the sinc function goes to 1 and the equation reduces to

$$
I_{2 \omega}(L)=2\left(\frac{\mu_{0}}{\epsilon_{0}}\right)^{\frac{3}{2}} \frac{(\omega d L)^{2}}{n_{\omega}^{2} n_{2 \omega}} I_{\omega}^{2}
$$

Although it is possible to get high conversion efficiencies with SHG in crystals, it can be difficult, so this model is sufficient for understanding the nonlinear behavior [34-37]. At high enough intensities, the pump energy will deplete and the frequency-doubled output will start to diminish and will eventually saturate. It is also important to note that, due to the phase matching condition, the polarization of the second-harmonic frequency will typically be orthogonal to the polarization of the pump frequency. For Type 0 phase matching, described in more detail in Section 2.4, the phase matching is not achieved via anisotropy but instead by modal phase matching, quasiphase matching, or dispersion engineering, and the second harmonic frequency can have the same polarization as the pump frequency [38]. Finally, we note that the modal overlap of the pump and SHG frequencies is important for SHG (or any second-order process) to occur. Mode overlap is trivial for beams within bulk nonlinear materials, but is an important consideration for nanophotonic devices [39, 40].

Second-order nonlinear effects, and especially SHG, are frequently used for converting IR light sources to UVVis range. For example, common green laser pointers are often made by frequency-doubling a $1064 \mathrm{~nm}$ wavelength source to $532 \mathrm{~nm}$ light using a nonlinear crystal. Although nonlinear conversion efficiencies are low, this scheme is often more efficient than creating a laser that emits at $532 \mathrm{~nm}$ directly due to the high efficiency of IR lasers, particularly those that emit at $1064 \mathrm{~nm}$. 


\section{$2.3 x^{(3)}$ effects: third harmonic generation and four wave mixing}

In the previous section, we considered the second-order term in the polarization power series (Eq. (6)). In this section, we consider the third-order term, $\chi^{(3)}$. Instead of considering three-wave mixing, we now consider fourwave mixing interactions in a nonlinear medium. In general, one can analyze four separate wavelengths mixing (Figure 2a). For simplicity, one will often analyze the degenerate case where $\omega_{1}=\omega_{2}=\omega_{3}=\omega_{4}=\omega$. We can generate four coupled-wave equations which can be useful for phase-conjugate mirrors, but not for upconverting IR light. However, the treatment extends to third-harmonic generation (THG), which is analogous to second-harmonic generation.

In THG, three incident photons of frequency $\omega$ are destroyed to create a single photon at frequency $3 \omega$ (Figure 2b). This process is useful for upconverting IR light into the visible, or more often UV range. We can follow a similar analysis for SHG and calculate the THG intensity $\left(I_{3 \omega}\right)$ :

$$
I_{3 \omega}(L)=\frac{\left(3 \omega \chi^{(3)}\right)^{2}}{16 \epsilon_{0}^{2} c^{4} n_{3 \omega} n_{\omega}^{3}} L^{2} I_{\omega}^{3} \operatorname{sinc}^{2}\left[\frac{\Delta k L}{2}\right]
$$

where $I_{\omega}$ is the fundamental beam intensity and $L$ is the distance propagated through the material. We see again the presence of a sinc function dependent on $\Delta k$, meaning we have similar phase-matching concerns as with secondorder nonlinearities to avoid problematic power cycling. In bulk materials, phase matching is often more difficult for THG as the material is not always anisotropic. The availability of other phase matching techniques, such as modal

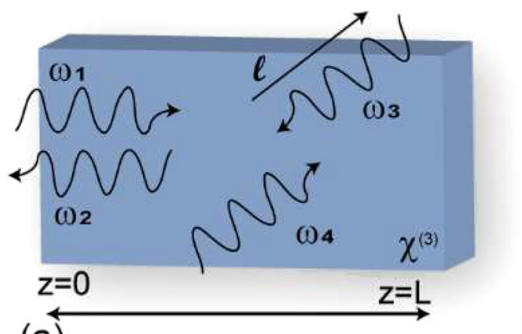

(a)

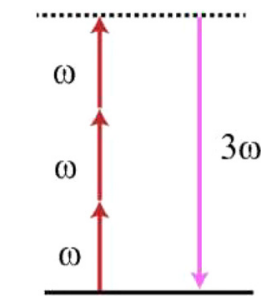

(b)
Figure 2: (a) Four waves with frequencies $\omega_{1}, \omega_{2}, \omega_{3}$, and $\omega_{3}$ mix in a nonlinear optical crystal exhibiting third-order $\left(x^{(3)}\right)$ effects, with two waves propagating in the $z$-direction and one propagating along vector $\ell$. (b) For third-harmonic generation (THG), three photons at the pump frequency are destroyed while interacting with the material to produce a single photon at triple the pump frequency $(1 / 3$ the wavelength). In THG, the incident (pump) photons and thirdharmonic photons can propagate in the same direction. phase matching, make nanophotonics an ideal platform for studying THG and other $\chi^{(3)}$ effects. Just as with secondorder effects, the third-harmonic and fundamental frequencies must have modal overlap with the nonlinear material and each other in order for THG to take place.

Practically speaking, THG via $\chi^{(3)}$ effects is inefficient. In many cases of THG, a second harmonic is generated via $\chi^{(2)}$ which then mixes with the unconverted frequency in another $\chi^{(2)}$ interaction (sum-frequency generation), producing the third harmonic, which tends to be more efficient than the $\chi^{(3)}$ process by itself. However, both have been used to demonstrate upconversion, as shown in references [37, 41-46]. Additionally, there are some important applications of $\chi^{(3)}$ processes, such as the generation of frequency combs [17, 47-55]. A frequency comb is a source with a spectrum that contains a series of equally-spaced frequency lines.

Another $\chi^{(3)}$ effect is the Kerr effect, in which highintensity light induces a refractive index change in the material (given by $n=n_{0}+n_{2} I$ ), which results in self-phase modulation. The Kerr effect contributes to other $\chi^{(3)}$ effects, such as dispersive wave generation, which is the result of soliton dynamics. In dispersive wave generation, a soliton propagating with a frequency distance $\delta \omega$ from the zerodispersion wavelength coherently couples to an optical wave with a frequency distance $-2 \delta \omega$ from the zerodispersion wavelength, which will be in-phase with the soliton. The zero-dispersion wavelength is the wavelength at which the material dispersion and modal dispersion offset one another. Dispersive wave generation can be analyzed as a cascaded four-wave mixing process [56].

Two-photon absorption (TPA) is a nonlinear process that occurs in a material where a single photon does not have enough energy to span the gap between a ground state and an excited state. At high enough intensities and when the energy gap to the excited state is equivalent to the energy of two photons, both photons can be simultaneously absorbed by the material, resulting in TPA. It is important to note that TPA is distinct from SHG, as SHG involves the conversion of two photons to a higherfrequency photon and TPA involves the absorption of two photons into a real excited state of the material. TPA can be modeled as a $\chi^{(3)}$ process using the following differential equation

$$
\frac{d I}{d z}=-\alpha I-\beta I^{2}
$$

where $\alpha$ is the linear loss and $\beta$ is the two-photon absorption coefficient. At the high intensities needed to drive other nonlinear processes, TPA can be a significant source of loss, especially in the UV-Vis range. 


\subsection{Phase matching in nonlinear materials (and quasi-phase-matching)}

Phase matching for $\chi^{(2)}$ processes is conventionally achieved by using an anisotropic crystal where you can have different refractive indices based on polarization. Because $\chi^{(2)}$ nonlinearities are only available in non-centrosymmetric crystals, they can take advantage of this anisotropy. Fewer $\chi^{(3)}$ materials are anisotropic so phase matching is often achieved by other means. Depending on the nonlinear crystal, there are two ways to achieve phase matching in uniaxial crystals, as shown in Table 1. In a practical sense, this means that for SHG and THG using this phase matching, the upconverted wavelength will be at a different polarization than the pump wavelength.

When phase matching may not be possible (as in a nonlinear waveguide), quasi-phase matching is often employed. In this method, the optical axis of the nonlinear material is made to alternate at regular intervals (Figure 3). This eliminates the problem of power cycling by inverting the phase with respect to the axis at a specified period, $\Lambda$. Creating the periodic optic axis in the structure is usually achieved via poling the material: using a very high electric field to force the axis to align with the electric field lines [57].

While phase matching is important for nonlinear interactions, particularly SHG and THG, it is also important to note that for thin films (short interaction lengths) it is less crucial. In integrated optical systems where there is some other condition imposed on the orientation of the crystal or isotropic nonlinear materials are used, phase matching

Table 1: Strategies to achieve phase matching in uniaxial crystals.

\begin{tabular}{lll}
\hline & Positive uniaxial $\left(n_{e}>n_{o}\right)$ & Negative uniaxial $\left(n_{e}<n_{o}\right)$ \\
\hline Type I & $n_{3}^{o} \omega_{3}=n_{1}^{e} \omega_{1}+n_{2}^{e} \omega_{2}$ & $n_{3}^{e} \omega_{3}=n_{1}^{o} \omega_{1}+n_{2}^{o} \omega_{2}$ \\
Type II & $n_{3}^{o} \omega_{3}=n_{1}^{o} \omega_{1}+n_{2}^{e} \omega_{2}$ & $n_{3}^{e} \omega_{3}=n_{1}^{e} \omega_{1}+n_{2}^{o} \omega_{2}$ \\
\hline
\end{tabular}

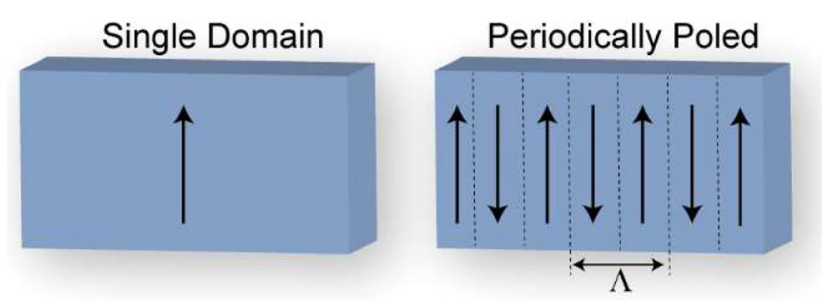

Figure 3: A crystal with a single domain (left) will have a single optical axis. With periodic poling (right) the crystal will have alternating optic axis with a period of $\Lambda$. Periodically-poled materials are frequently used for phase-matching in nonlinear interactions. conditions must often be met using other strategies. These other phase matching conditions (including quasi-phase matching) are often referred to as Type 0 phase matching. One example is using modal dispersion to phase-match, which may depend on material choices and/or the specific geometry of the device $[58,59]$. In modal dispersion phase matching, the device is designed such that the effective refractive index of the modes supporting the pump and generated (second-harmonic or third-harmonic) frequencies match. Additionally, the dispersion of the light signal may be engineered (via the use of metamaterials or photonic crystals, for example) in order to ensure phase matching conditions are met. These other phase matching techniques are particularly important, because in an integrated device, it is often not possible to rotate the nonlinear crystal axes with respect to the optical field once the device has been fabricated, and isotropic nonlinear materials are attractive for applications in the UV-Vis range.

\subsection{Other effects: Raman scattering, Brillouin scattering, supercontinuum}

Raman scattering is a process where a photon traveling through a material loses energy by exciting vibrational states of the material or gains energy from those same vibrational states. In a typical Raman process, energy from the photon is lost to the material vibrational modes, creating a longer wavelength referred to as a Stokes wavelength or Stokes shift. Once vibrational modes are resonating, subsequent pump photons may pick up energy from the vibrational mode, creating an anti-Stokes wavelength or anti-Stokes shift (Figure 4). The relationship of scattered power $\left(\mathrm{P}_{\text {scatter }}\right)$ to the incident intensity is characterized by a scattering cross-section $\left(\sigma_{R}\right)$, which is typically measured experimentally:

$$
P_{\text {scatter }}=\sigma_{R} I_{0}
$$

where $I_{0}$ is the incident intensity. Typically, Stokes scattering alone is not useful for upconverting IR to UV or visible light (it downconverts or redshifts the wavelength), but anti-Stokes scattering (which occurs in conjunction with Stokes scattering) can be used for upconversion. This is typically efficient in systems where there is a very large intensity, such as high-Q resonators where photons circulate with a very long lifetime, or high confinement, as in plasmonic nanoparticles or structures [60-64].

Stimulated Raman scattering is a process where the pump and Stokes wavelengths are introduced into the material to generate an enhanced Raman response where energy is transferred from the pump wavelength to the 


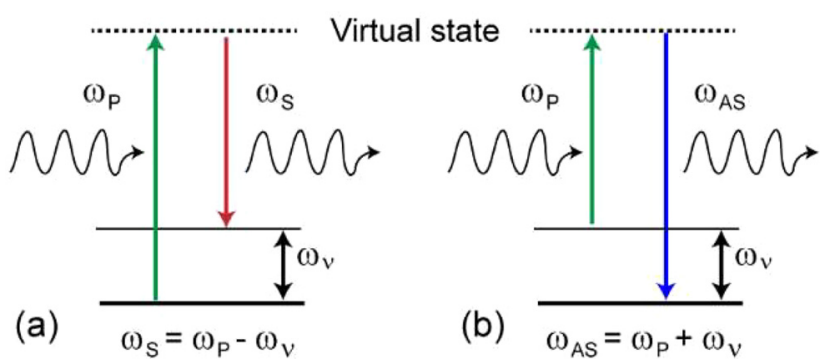

Figure 4: Stimulated Raman scattering can generate Stokes and anti-Stokes frequencies. (a) For a Stokes shift, energy from the incident photon is lost to vibrational modes of the material and a lower frequency (longer wavelength) photon is scattered. (b) For an anti-Stokes shift, the incident photon gains energy from the vibrational modes of the material and the scattered photon has a higher frequency (shorter wavelength).

Stokes wavelength. The interactions from stimulated Raman scattering are typically analyzed as $\chi^{(3)}$ processes with the Stokes intensity given by:

$$
I_{S}(L)=I_{S}(0) e^{g_{R} I_{P} L}
$$

where $g_{R}$ is the Raman gain intensity factor, $I_{S}$ is the Stokes beam intensity, $I_{P}$ is the pump beam intensity, and $L$ is the propagation length for the undepleted pump approximation. The Raman gain intensity factor is often measured experimentally, although it can be defined in terms of refractive indices for the pump and Stokes beam, the Stokes frequency and the imaginary part of $\chi_{R}{ }^{(3)}$, the Raman susceptibility. Because the pump and Stokes beams are coupled via the vibrational modes in the material, Raman scattering is always phase-matched in the propagation direction of the pump beam.

As opposed to exciting vibrational states of the material (Raman scattering), photons may also excite acoustic waves within a material, which will generate periodic refractive index variations (the refractive index depends on density). If a photon is subsequently scattered off this acoustic wave, the result is Brillouin scattering. Stimulated Brillouin scattering is a process where a spontaneously scattered photon with a frequency difference from the pump equal to the frequency of the acoustic wave couples with the pump beam to drive the acoustic wave. This most often occurs when the photon scatters in the backward direction and can be a limiting factor in fiber optic communications. Stimulated Brillouin scattering has recently seen much interest in the integrated photonics community, and it is discussed in more detail in reference [65].

One important application of nonlinear phenomena is the generation of supercontinua. A supercontinuum is a light signal with a broad and smooth spectrum. They are generated by multiple nonlinear processes interacting, such as four-wave mixing, Raman scattering, sum-frequency generation, dispersive wave generation, and self-phase modulation (a process where light intensity induces a refractive index change in the material, leading to a chirped pulse). The specific nonlinear effects used for generating a supercontinuum will depend on the materials being used and occasionally on the geometry of the device used for generation. Supercontinuum generation has been used across the visible and UV range in nanophotonic devices [66, 67].

\section{Nonlinear optical materials}

\subsection{Materials overview}

In the past a few years, photonics based on narrow bandgap semiconductors such as $\mathrm{Si}$ and GaAs III-V have achieved great success in nanophotonic devices as well as integrated photonics circuits (PICs). However, the narrow bandgap energy of these materials, e.g., $1.1 \mathrm{eV}$ of $\mathrm{Si}$, has restricted the light transmission to wavelengths longer than $1130 \mathrm{~nm}$, which has hindered their applications in UV-Vis spectral region. Alternatively, wide bandgap semiconductor/dielectric materials, such as silica, SiN, $\mathrm{SiC}$, diamond, lithium niobate, and III-N (e.g., GaN and AlN), have received considerable attention for the nanophotonic applications in the UV-Vis range.

Silicon dioxide (or silica) possesses the largest bandgap energy among these materials, thus exhibiting extreme broadband transparency in the visible through the near-IR. Additionally, over the past several decades, numerous wet chemistry and deposition routes have been developed for making both undoped and doped silica layers [68-72]. When combined with the ease of processing and the compatibility of silica with common microprocessing methods, silica has formed the foundation for a plethora of technologies [21, 73-81]. However, in the context of nonlinear performance, one significant limitation of silica is its low nonlinear behavior. Unless in the form of quartz (which is rare in an integrated platform), silica is an amorphous material, lacking inherent symmetry. While methods like high temperature or thermal poling can be used to induce an electro-optic $\left(\chi^{(2)}\right)$ response, it is adds complexity to fabrication [82]. However, due to the exceptionally low optical loss of silica, it is possible to fabricate devices allowing optical field amplification without damaging the material, allowing nonlinear behaviors to be excited [83-93]. In addition, the ease of doping the amorphous silica matrix provides a complementary route to designing and realizing nonlinear devices from silica [18, 77, 84, 94-99]. 
Silicon nitride is a popular wide bandgap dielectric material that has been the focus of numerous research efforts. The measured single mode propagation loss for silicon nitride is below $1 \mathrm{~dB} / \mathrm{cm}$ in the $532-900 \mathrm{~nm}$ range [100]. Due to the central-symmetric crystalline quality, its $\chi^{(2)}$ nonlinearity is zero except under the presence of interface strain [58] and strong bias [101]. The $\chi^{(3)}$ nonlinearity of silicon nitride in the form of Kerr refractive index $\left(n_{2}\right)$ was identified to be $2.5 \times 10^{-15} \mathrm{~cm}^{2} / \mathrm{W}$ at $1550 \mathrm{~nm}$ by evaluating the nonlinear optical response of an optical resonator [102]. It was also reported that the nonlinear response can be further enhanced by 5 times if silicon-rich silicon nitride waveguides were adapted [103]. One of the major advantages of silicon nitride platform is its excellent CMOS compatibility [104, 105], which provides the potential for multi-layer [106] and high-density integration [107].

In addition to silica and silicon nitride, several other materials have been studied for nonlinear applications in the visible spectrum. For example, lithium niobate has been extensively investigated due to its strong $\chi^{(2)}$ on the order of $d_{33}=-20.6 \mathrm{pm} / \mathrm{V}$ [108] and the significant electro-optic effect [109]. Recent developments on Lithium-niobate-oninsulator (LNOI) technology $[110,111]$ showed high potential for its adaption in integrated photonic applications. An ultra-low loss of $6 \mathrm{~dB} / \mathrm{m}$ at $637 \mathrm{~nm}$ has been achieved [110] in a lithium niobate waveguide with visible electro-optic bandwidth exceeding $10 \mathrm{GHz}$. In addition, a recent work showed that $4 \mathrm{H}$-silicon-carbide-on-insulator (4H-SiCOI) technologies [112] offer unique optical properties for quantum photonic and nonlinear optical applications, where a relatively high second order nonlinearity of $d_{33}=-12.5 \mathrm{pm} / \mathrm{V}$ at $1.064 \mu \mathrm{m}$ [113] is observed. This is promising for efficient parametric conversions. However, further investigation is still required for this new platform. Another promising material is diamond. It has excellent optical properties for quantum emitters [114] and PICs [115], and it has been proposed for wide variety of nonlinear optical applications. Atomic layer deposited $\mathrm{Al}_{2} \mathrm{O}_{3}$ was also reported with excellent wave guiding behavior in the UV spectrum with propagation loss of $\langle 3 \mathrm{~dB} / \mathrm{cm}$ at $371 \mathrm{~nm}$ [116]; however, it has a relatively weak modal confinement and a small Kerr refractive index [117]. AlGaAs possesses strong third order nonlinearities $\left(n_{2}=2.6 \times 10^{-13} \mathrm{~cm}^{2} / \mathrm{W}\right)$ over most popular dielectric materials, which leads to Kerr comb generation with extreme low threshold values [118]. Comprehensive reviews and representative demonstrations on aforementioned materials can be found in the prior references. Due to the space limit, we focus our discussions on GaN-based III-N and organic materials, which have emerged as two groups of exciting new materials for nonlinear integrated photonics applications particularly for UV-Vis wavelength range.

\subsection{III-N materials}

The overall advantages of III-N materials originate from their wide bandgap energies, outstanding properties in both optoelectronics and nonlinear optics, and excellent compatibility with existing III-N light sources in the UVVis spectral range. The properties of GaN includes a wide bandgap energy of $3.4 \mathrm{eV}$, corresponding to a transparent wavelength above $365 \mathrm{~nm}$. Due to the close lattice constant with InGaN LEDs/lasers [119] and AlGaN RF electronic components [120], GaN is promising for active optoelectronic integration. By Maker-Fringe measurements, the quadratic nonlinear-optical coefficient of $\mathrm{GaN}$ was identified to be $d_{31}=2.5 \pm 0.1 \mathrm{pm} / \mathrm{V}$ and $d_{33}=-3.8 \pm 0.1 \mathrm{pm} /$ $\mathrm{V}$ [121]. Some early characterizations on nonlinear optical properties of GaN layers on sapphire substrates (i.e., typically with defect density of $>10^{9} \mathrm{~cm}^{-2}$ ) using the Z-scan method suggests a two-photon absorption (TPA) coefficient of $\beta=17 \pm 7 \mathrm{~cm} / \mathrm{GW}$ at $\lambda=400 \mathrm{~nm}$ and $\beta=3 \pm 1.5 \mathrm{~cm} / \mathrm{GW}$ at $\lambda=720 \mathrm{~nm}$ [122]. In contrast, recent results on $\mathrm{GaN}$ bulk materials (i.e., with threading dislocation defect density of $<10^{6} \mathrm{~cm}^{-2}$ ) reveal that the intrinsic TPA coefficient is about $0.9 \mathrm{~cm} / \mathrm{GW}$ at $724 \mathrm{~nm}$ [123] and $3.5 \mathrm{~cm} / \mathrm{GW}$ at $532 \mathrm{~nm}$ [124]. The improvements in the TPA coefficients on GaN bulk materials can be attributed to improved material quality, by which the density of deep level defects [125] and n-type donors (nitrogen vacancies) [126] are greatly reduced compared to GaN layers on sapphire substrates. The Kerr refractive indexes $\left(n_{2}\right)$ of GaN were identified to be $n_{2}=1.15-1.4 \times 10^{-14} \mathrm{~cm}^{2} \mathrm{~W}^{-1}$ at $\lambda=800 \mathrm{~nm}$ [127]. However, because the growth of $\mathrm{GaN}$ is usually accompanied with large amount of threading dislocations, the two-photon absorption coefficient of $\mathrm{GaN}$ is relatively large in comparison with other wide bandgap semiconductors [128].

Compared to GaN, AlN has an even wider bandgap energy of $6 \mathrm{eV}$, allowing broadband transparency from deep-UV to infrared. The quadratic nonlinear optical coefficient of AlN was characterized by A. Majkić et al. [129]. This work measured an absolute value of $d_{33}=4.3 \pm 0.3 \mathrm{pm} /$ $\mathrm{V}$ at $\lambda=1030 \mathrm{~nm}$, and the $d_{31}$ was in the range of $d_{33} /(45 \pm 5)$. Since the AlN is mainly grown on $c$-plane of sapphire substrate, the measured $d_{33}$ and $d_{31}$ suggests that TM modes possess a larger second order nonlinearity, which will lead to a higher conversion efficiency in second harmonic generation (SHG) [130] and a degenerate parametric down conversion [131]. Due to the difficulties in obtaining high quality bulk AlN and the large two-photon absorptive photon energy, direct Z-scan measurement on high quality AlN is still not reported. One exception is the report by M. Zhao et al. [132], in which the TPA coefficient and $n_{2}$ at 
$\lambda=355 \mathrm{~nm}$ were measured to be $13 \pm 3 \mathrm{~cm} / \mathrm{GW}$ and $-1.91 \pm 0.38 \times 10^{-13} \mathrm{~cm}^{2} \mathrm{~W}^{-1}$, respectively. Noting that the AlN sample used in the previous work [132] exhibits strong optical absorption below $\lambda=300 \mathrm{~nm}$, possibly due to the inferior crystalline quality of the AlN. Alternatively, one can also adopt the derived $n_{2}$ of $2.3 \pm 1.5 \times 10^{-15} \mathrm{~cm}^{2} \mathrm{~W}^{-1}$ at $1550 \mathrm{~nm}$ for AlN [133], and utilize the wavelength dependence fitting provided in previous work [134] to roughly estimate the nonlinear optical performance of AlN in the $\mathrm{UV}$ and visible spectral range. The estimated $n_{2}$ at $800 \mathrm{~nm}$ is $3 \times 10^{-15}$ and $1 \times 10^{-14} \mathrm{~cm}^{2} \mathrm{~W}^{-1}$ at $400 \mathrm{~nm}$.

\subsection{Organic materials}

While initial work in the field of organic photonics focused on polymers $[145,146]$, organic small molecules are emerging as an alternative material for a wide range of applications [147-153]. Organic nonlinear optical small molecules are $\pi$-conjugated molecules, and the strong nonlinear optical susceptibilities and rapid responses are based on highly movable $\pi$-electrons along the molecular backbone [154-158]. While the timescales of the nonlinear response are comparable, the magnitudes that can be achieved are larger than in conventional crystalline systems, enabling higher performing devices. In addition, the nanometer-scale length or size of the material needed to achieve a nonlinear response is much smaller than either conventional crystalline materials or organic polymers. However, the response is dependent on the molecular orientation of the molecule.

Specifically, the nonlinear optical response of an organic molecule that is induced can be described by the expression below [159]:

$$
\mu_{i}=\alpha_{i j} E_{j}+\beta_{i j k} E_{j} E_{k}+\gamma_{i j k l} E_{j} E_{k} E_{l}+\ldots
$$

where $\mu_{\mathrm{i}}$ is the induced dipole moment in a molecule, $\mathrm{E}_{\mathrm{i}}$ is the electromagnetic field, $\alpha, \beta$, and $\gamma$ are the linear polarizability, first hyperpolarizability, and second hyperpolarizability tensors, respectively. It is important to note that this expression describes the efficiency of charge transfer $(\beta)$ or vibration $(\gamma)$ at the molecular level [160]. The $\beta_{\mathrm{ijk}}$ is responsible for second order nonlinear effects, and the $\gamma_{\mathrm{ijkl}}$ is responsible for third order nonlinear effects, as described by the macroscopic susceptibilities $\chi^{(2)}$ and $\chi^{(3)}$ in Eq. (6). However, it is important to note that the macroscopic susceptibilities are related, but not equal, to the molecular nonlinearities. Several factors influence this relationship including the density of the organic molecules and the orientation of the molecule with the incident optical field. As one might imagine, there are nearly limitless possible organic chemical structures that could be synthesized that would exhibit nonlinear behavior.

For comparison, Table 3 lists $n_{2}$ of a variety of inorganic and organic materials mentioned in this review. In inorganic materials conventionally used to fabricate integrated optical circuits, $n_{2}$ follows the range of $\sim 10^{-18}$ to $\sim 10^{-20} \mathrm{~m}^{2} / \mathrm{W}$. Organic materials show a higher magnitude but similar variance of $n_{2}$ values. However, one challenge when making a table is determining the absolute value to report. As mentioned, the $n_{2}$ depends on the molecular density and molecular orientation with the optical field.

To limit the scope of this article, we will focus on the two different chemical structures shown in Figure 5: Tetraphenylethylene (TPE) and 4-[4-diethylamino(styryl)] pyridinium (DASP).

TPE is an intriguing material with a unique chemical structure. In previous work, it has demonstrated large NLO coefficients, particularly the first hyperpolarizability or the second-order NLO coefficient, and the four-leaf clover architecture facilitates the design of push-pull chromophores that allows electron charge transfer across the $\pi$ conjugation of the TPE molecule [170-179]. It has previously been used successfully as an imaging agent $[155,155,180]$, and,

Table 2: Nonlinear optical properties of III-N in comparison with other materials.

\begin{tabular}{|c|c|c|c|c|c|}
\hline Material & Optical bandgap (eV) & Refractive index & Dominant $d_{i i}(p m / V)$ & $n_{2}\left(\mathrm{~cm}^{2} / \mathrm{W}\right)$ & Linear loss in visible $(\mathrm{dB} / \mathrm{cm})$ \\
\hline $\mathrm{SiO}_{2}$ & 8.9 & 1.45 & & $4 \times 10^{-16}[135]$ & 0.97 (at $658 \mathrm{~nm}$ ) [136] \\
\hline $\mathrm{Si}_{3} \mathrm{~N}_{4}$ & 5.1 & 2.05 & - & $2.5 \times 10^{-15}[102]$ & $<1(532 \sim 900 \mathrm{~nm})[100]$ \\
\hline $\mathrm{SiC}$ & $2.3 \sim 3.3$ & 2.6 & $-12.5[113]$ & $1.9 \pm 0.7 \times 10^{-15}[137]$ & $2.3($ at $633 \mathrm{~nm})$ [138] \\
\hline $\mathrm{Al}_{2} \mathrm{O}_{3}$ & 9.1 & 1.77 & - & $(3.3 \sim 2.8) \times 10^{-16}[117]$ & $<2$ (at $405 \mathrm{~nm}$ ) [116] \\
\hline Diamond & 5.5 & 2.42 & - & $1.3 \times 10^{-15}[115]$ & \\
\hline AlGaAs & $1.4 \sim 2.2$ & $2.9 \sim 3.4$ & 90 [139] & $2.6 \times 10^{-13}[118]$ & \\
\hline $\mathrm{TiO}_{2}$ & $3 \sim 3.5$ & 2.4 & - & $2.3 \sim 3.6 \times 10^{-14}[140]$ & $28($ at $635 \mathrm{~nm})[141]$ \\
\hline $\mathrm{LiNbO}_{3}$ & 3.7 & 2.2 & $-20.6[108]$ & $5.3 \times 10^{-15}[142]$ & $0.06($ at $637 \mathrm{~nm})[110]$ \\
\hline GaN & 3.4 & 2.4 & $-3.8 \pm 0.1[121]$ & $1.15 \sim 1.4 \times 10^{-14}[127]$ & $\sim 2$ (at $700 \mathrm{~nm})[143]$ \\
\hline AIN & 6.2 & 2.16 & $4.3 \pm 0.3[129]$ & $2.3 \pm 1.5 \times 10^{-15}[133]$ & $3 \pm 1($ at $635 \mathrm{~nm})[144]$ \\
\hline
\end{tabular}


Table 3: Nonlinear optical properties of several commonly used organic materials.

\begin{tabular}{|c|c|c|c|c|c|}
\hline Material & Structure & Optical bandgap (eV) & Refractive index & First-order hyperpolarizability (m/V) & $n_{2}\left(\mathrm{~cm}^{2} / \mathrm{W}\right)$ \\
\hline DASP & & $3.9 * \star$ & 1.57 & $(2.6 \sim 3.7) \times 10^{-32}[161]$ & $2.5 \times 10^{-13}[162]$ \\
\hline TPE & & $3.6[163]^{*}$ & 1.64 & $3.1 \times 10^{-33}[163]^{\star}$ & $1.9 \times 10^{-15}[164]^{\star}$ \\
\hline Anthracene & & $3.1[165]$ & 1.59 & $2.2 \times 10^{-37}[166]$ & $2.6 \times 10^{-15}[167]$ \\
\hline PTCDA & & $2.1[168]$ & 2.21 & - & $1.2 \times 10^{-13}[169]$ \\
\hline
\end{tabular}

*Values of derivative structures. ** Calculated by time-dependent density functional theory with the gas phase ground state molecular geometry optimized at the B3LYP/6-31G* level of theory.

(a)<smiles>c1ccc(C(=C(c2ccccc2)c2ccccc2)c2ccccc2)cc1</smiles>

(c)<smiles>CCN(CC)c1ccc(/N=N/c2ccccc2)cc1</smiles>

(b)<smiles>[R]c1ccc(C(=C(c2ccccc2)c2ccccc2)c2ccc([R])cc2)cc1</smiles>

(d)

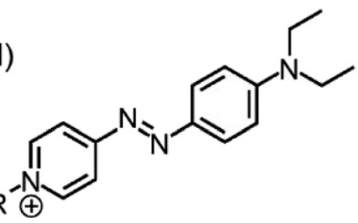

Figure 5: Chemical structures of commonly used organic small molecules. (a) TPE and (b) TPE with common sites for modification indicated by $R$ and $R^{\prime}$. (c) DASP and (d) DASP with common sites for modification indicated by $R$.

in contrast to many fluorophores, it is well-known for exhibiting aggregation-induced-emission (AIE) [171, 181] in which the emission intensity increases as the molecules aggregate.

One study measured the nonlinear optical activity of a series of TPE structures (Figure 6) [170]. In this study of eight different structures, the second-order NLO polarizabilities was increased as much as 27 times by tuning the substituent groups and their positions even when the primary chemical composition (the TPE core) was relatively unchanged. As can be seen in Figure 6, Structure 1 starts with hydrogens in all $\mathrm{R}_{1}-\mathrm{R}_{4}$ substituent groups. In this case, the electron density distribution of Structure 1 from the density functional theory (DFT) calculation is fairly

uniform (Figure 6b, inset), meaning that the intramolecular charge transfer (ICT) is less likely to occur. In contrast, in Structure 8 , the $R_{1}$ and $R_{4}$ were replaced by the donor Ph$\mathrm{NH}_{2}$, and $\mathrm{R}_{2}$ and $\mathrm{R}_{3}$ were replaced by the acceptor $\mathrm{Ph}-\mathrm{NO}_{2}$. In this molecule, the electron density distribution is highly asymmetric due to the asymmetric orientation of the donoracceptor R groups (Figure 6b, inset). It is important to note that this work focused on tuning the conjugation length via $\mathrm{NH}_{2}$ and $\mathrm{NO}_{2}$ groups. However, there are numerous other strategies that have also been employed successfully, such as bromination.

In addition to purely synthetic routes, researchers have also employed predictive methods. These have been particularly successful in accelerating material design for small organic molecules which have fewer atoms than larger polymeric systems. Initial approaches, such as DFT, were based on quantum mechanical calculations of the orbital structures [182-185]. Hydrogen bond pattern prediction based on similar quantum mechanical modeling has also been successful in the design and prediction of NLO organic molecules. Freely available shareware has popularized these methods among chemists and material scientists impacting material design in a range of fields [186-194]. More recent computational methods have relied on utilizing an algorithm which can quickly find minimums in the potential energy landscapes of novel organic materials.

The chemical structures of 4-[4-diethylamino(styryl)] pyridine (DASPy) and 4-[4-diethylamino(styryl)]pyridinium (DASP) are depicted in Figure 5. When the pyridine moiety is substituted with a R group, DASPy becomes its pyridinium form DASP. As seen, there is one equivalent of 


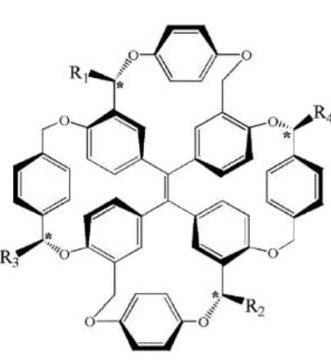

(a)
1: $\mathrm{R}_{1}=\mathrm{R}_{2}=\mathrm{R}_{3}=\mathrm{R}_{4}=\mathrm{H}$

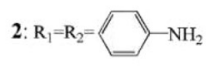

$\mathrm{R}_{3}=\mathrm{R}_{4}=\mathrm{H}$

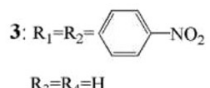

$\mathrm{R}_{3}=\mathrm{R}_{4}=\mathrm{H}$
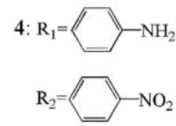

$\mathrm{R}_{3}=\mathrm{R}_{4}=\mathrm{H}$

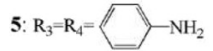

$\mathrm{R}_{1}=\mathrm{R}_{2}=\mathrm{H}$

6: $\mathrm{R}_{3}=\mathrm{R}_{4}=\square-\mathrm{NO}_{2}$

$\mathrm{R}_{1}=\mathrm{R}_{2}=\mathrm{H}$

7: $\mathrm{R}_{3}=\square-\mathrm{NO}_{2}$

$\mathrm{R}_{4}=\square-\mathrm{NH}_{2}$

$\mathbf{R}_{1}=\mathbf{R}_{2}=\mathrm{H}$

8: $\mathrm{R}_{1}=\mathrm{R}_{4}=\square-\mathrm{NH}_{2}$

$\mathrm{R}_{2}=\mathrm{R}_{3}=\square-\mathrm{NO}_{2}$

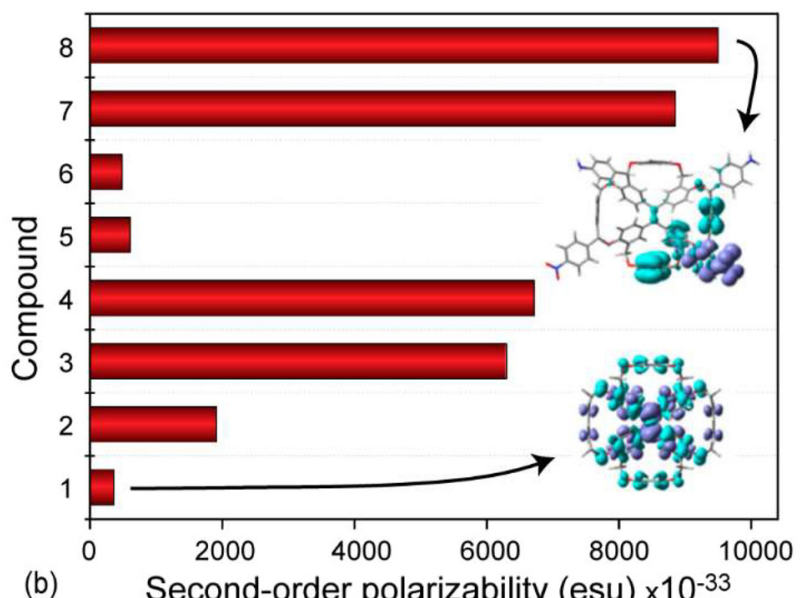

(b) Second-order polarizability (esu) $\times 10^{-33}$

Figure 6: (a) Structures of a series of cyclic TPEs. (b) Second-order polarizabilities of the synthesized TPE derivatives in part (a). The second order polarizability increased approximately 27 times from $1.50 \times 10^{-34} \mathrm{~m} / \mathrm{V}$ to $3.98 \times 10^{-33} \mathrm{~m} / \mathrm{V}$ (converted from esu units) by changing the residues. Inset: DFT modeling of compounds 1 and 8. Adapted with permission from C. Liu, G. Yang, Y. Si, X. Pan, Journal of Physical Chemistry C. 122(9):5032-39 (2018). Copyright 2018 American Chemical Society.

positive charge on pyridinium in DASP compared to DASPy, and this additional formal charge makes DASP a "push-pull" chromophore where electron-rich amine group favors to donate electrons to electron-poor pyridinium [195-198]. Thus, as long as DASP molecules can be functionalized wisely on a specific surface, such delocalization of electrons on DASP makes it suitable for photoactive materials in NLO studies.

Over two decades ago, scientists began investigating the SHG behavior in DASP Langmuir-Blodgett (LB) films. To further improve the nonlinearity, one reported a series of rare Earth metal dysprosium-DASP complexes [199, 200]. According to the authors, dysprosium complexes serving as the R group facilitate the formation of uniform LB films. They characterized the SHG with the Nd:YAG laser beam at with a peak power at $1064 \mathrm{~nm}$, anticipating SHG emissions at $532 \mathrm{~nm}$. Both of the compounds investigated achieved $\chi^{(2)}$ and $\beta$ values in the $10^{-6}$ and $10^{-27}$ esu range, respectively. Zhao et al. also discovered that the solvent environment has an effect on SHG performances of DASP LB films [201]. For example, when the vapor pressure of isopropanol increases, the SHG signal decreases. Even after the solvent vapor pressure is removed, the SHG signal is not fully recovered. Such considerations will play a key role when considering and optimizing processing conditions for integration with onchip optical devices.

\section{Device platform and applications}

\subsection{Device platforms}

The most straightforward device to fabricate on a silicon wafer is an integrated waveguide. The first on-chip waveguides were simple slab, or rectangular, waveguides with uniform indices [202]. These devices essentially mimicked optical fibers on-chip. Subsequently, researchers realized that nanofabrication opened up possibilities not easily available when drawing fibers, including index and geometric variations over nano-scale dimensions [203-205]. Such devices enable control over the material dispersion, and therefore, they form a key component in the engineer's toolbox, enabling nonlinear behaviors to be accessed.

A second commonly used device is the optical resonant cavity. Both traveling wave and standing wave resonators have been used in nonlinear optics investigations as both cavities are able to achieve the high optical intensities needed to unlock nonlinear behaviors [206]. Photonic crystal cavities, a common standing wave resonator, have small optical mode volumes which focus the light, resulting in high intensity [41]. In contrast, while whispering gallery mode cavities have large optical mode volumes, they can achieve high optical cavity quality factors (Q) due to their long photon lifetimes [83]. The high $Q$ values allow them to achieve large circulating intensities.

Initial work investigating nonlinear phenomena was in the near-IR and focused on leveraging the ultra-high-Q factors possible in the whispering gallery mode cavities, particularly in silica and fluoride devices [207]. Despite the low nonlinear coefficients, Stokes and Anti-Stokes behavior [208-210] as well as four wave mixing [21, 85, 88, 99, 211-213] were demonstrated. More recent efforts which are the focus of this section are investigating new material platforms to improve the nonlinear coefficient of the material and to open the door to new wavelength ranges. 


\subsection{III-N materials}

The major nonlinear optical application of $\mathrm{GaN}$ is the SHG from the telecommunications wavelengths $(\sim 1550 \mathrm{~nm})$ to the near-visible spectral. C. Xiong et al. reported the SHG from $\mathrm{GaN}$ ring resonators with measured quality factors around 10,000 at $\sim 1550 \mathrm{~nm}$ [214]. I. Roland et al. also reported the SHG at similar wavelengths using GaN-on-silicon suspended disk resonators [215], and the typical loaded $Q$ factors were in the range of 6000 to 13,000 . However, the typical $Q$ factors of $\mathrm{GaN}$ resonators are limited at $10^{4}$, which are more than two orders of magnitudes lower than the typically $Q$ values obtained from resonators based on other materials [216, 217] with similar dry etch fabrication methods. Recently, $\mathrm{H}$. Chen et al. investigated the loss mechanisms on GaN photonic devices and suggested that the nitrogen vacancy contributed n-type conductivity $\left(\mathrm{N}_{\mathrm{O}}=10^{18} \mathrm{~cm}^{-3}\right)$ was responsible for the high propagation loss in GaN devices, where a high free carrier absorption loss of $>2 \mathrm{~dB} / \mathrm{cm}$ be expected [143]. This high degree of free carrier absorption has hindered the development of high $Q$ resonators for $\mathrm{GaN}$ devices, which are typically epitaxially grown and fabricated on sapphire or silicon substrate and therefore contain high defect densities. It is noteworthy that the recent development of GaN devices fabricated on high quality GaN bulk substrates [134] could potentially tackle this challenge by reducing the density of nitrogen vacancies in the GaN materials. Further investigations on these new "GaN-on-GaN" devices are required to achieve high $Q$ GaN resonators.

Compared to GaN, the wider bandgap of AlN allows for a very low n-type conductivity, and consequently much lower free carrier absorption loss. As a result, extensive investigations have been carried out on AlN devices, where AlN ring resonators with very high $Q$ values have been demonstrated. In the telecommunications spectral region, the AlN ring resonators showed typical intrinsic $Q$ factors on the order of $10^{6}$ for both TE and TM modes [216, 218]. In the near-visible spectral region, $Y$. Sun et al. demonstrated AlN ring resonators with intrinsic $Q$ factors greater than 70,000 [219], while T. J. Lu et al. reported AlN devices with intrinsic $Q$ values exceeding 170,000 at $638 \mathrm{~nm}$ [220]. In the UV spectra region, $\mathrm{X}$. Liu et al. demonstrated AlN devices with intrinsic $Q$ factors of 210,000 [221]. AlGaN/AlN alloys were also theoretically proposed [222], in which the low index contrast is promising for low loss waveguiding. The relative higher $Q$ factors on AlN devices allows more efficient frequency conversion from telecommunications to near-visible spectrum [223] and vice versa [131] through the $\chi^{(2)}$ processes.
Despite the successful demonstration of high $Q$ resonators on AlN, the large chromatic dispersion and group velocity dispersion in the UV and Vis spectra remain to be the significant challenges towards realizing highly efficient frequency conversion through $\chi^{(2)}$ or $\chi^{(3)}$ process using AlN devices. Recently, X. Liu et al. proposed a chirp-modulated taper AlN waveguide to achieve broad phase matching using a near-visible pumping wavelength [221]. By fully utilizing the $\chi^{(2)}$ and $\chi^{(3)}$ nonlinearities, over $100 \mathrm{THz}$ of coherent frequency spanning (360-425 nm) was achieved on AlN, and the results are as shown in Figures $7 \mathrm{a}$ and $\mathrm{b}$. Alternatively, coherent generation in the UV and visible spectra can also be achieved using the soliton dynamics. For example, D. Y. Oh et al. successfully demonstrated the coherent generation in the UV wavelengths using dispersive wave generation from silica ridge waveguides [224], which is shown in Figure 7c. The dispersive waves were widely tunable from 322 to $545 \mathrm{~nm}$ by tuning the waveguide geometry. Excellent coherence was maintained since the frequency conversion is phase matched. More recently, $\mathrm{H}$. Chen et al. demonstrated that the same engineering principle can be applied to AlN devices, where supercontinuum generation from near UV-Vis spectra was achieved using dispersion engineered AlN waveguides (as shown in Figures $7 \mathrm{~d}$ and e) [225]. The AlN waveguides were pumped in $\mathrm{TE}_{10}$ mode near the zero dispersion wavelength (ZDW) at $810 \mathrm{~nm}[225]$. Due to the relatively large nonlinear parameter of AlN [221, 225] compared with silica [224], the pulse energy of AlN waveguides was significantly reduced to less than $1 \mathrm{~nJ}$ while covering a much broader spectral region, while the typical operation pulse energy within silica is above $1 \mathrm{~nJ}$ [224]. This work opens door for integrated AlN nanophotonic devices and circuits, which will lead to potential applications in on-chip mode locking [226], parametric oscillator [227], and entangled photon generation [228].

\subsection{Organic materials}

The fabrication of devices from nonlinear organic materials is intrinsically more complex than from III-V or II-IV materials due to their inherent incompatibility with the majority of nanofabrication procedures. In past work using organic polymeric materials, it was common to fabricate the entire device from the polymer using either nanoimprinting or laser writing methods [13, 229]. Using this approach, several different on-chip structures were fabricated including waveguides, resonators, and modulators with $\mathrm{GHz}$ rates (8-16). However, because the device is fabricated from the polymer, the optical performance is limited by the optical loss of the material which can be 


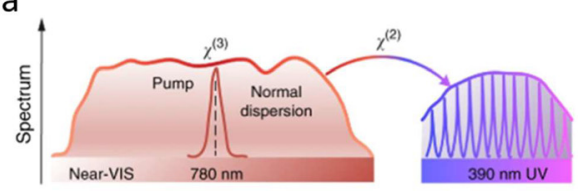

b

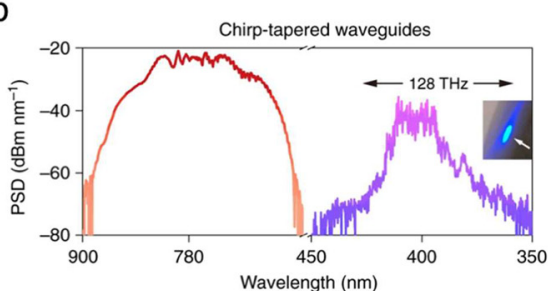

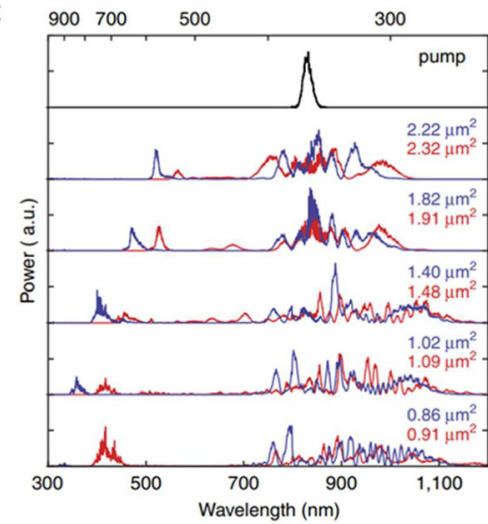

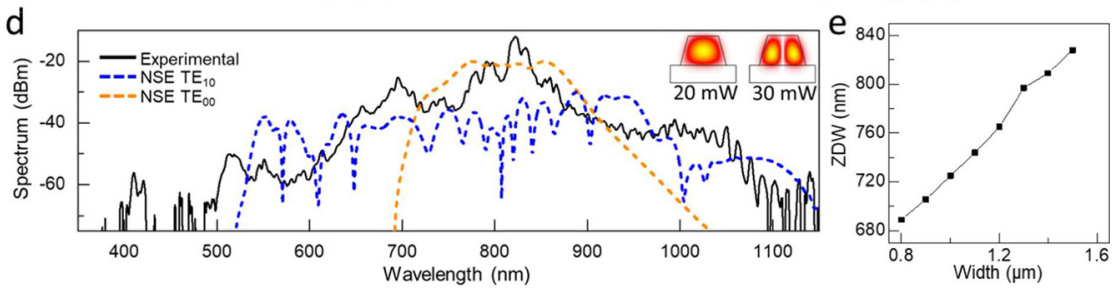

Figure 7: (a) The coherent generation in the UV spectra via $X^{(2)}$ and $\chi^{(3)}$ processes simultaneously from a chirp-modulated taper AIN waveguide. (b) The broad spectrum obtained from near-the chirp-tapered AIN waveguide using a near-visible pumping wavelength. Reprinted from [221]. Distributed under a Creative Commons Attribution NonCommercial License 4.0 (CC BY-NC) http://creativecommons.org/licenses/by$\mathrm{nc} / 4.0 /$ (c) The coherent dispersive wave generation in the UV and visible spectra through soliton dynamics using silica ridge waveguides. Red and blue curves indicate TE and TM mode operation, respectively. Reprinted from [224]. Distributed under a Creative Commons Attribution NonCommercial License 4.0 (CC BY-NC) http:// creativecommons.org/licenses/by-nc/4.0/ (d) The supercontinuum generation from AIN waveguide pumped near zero dispersion wavelength in $\mathrm{TE}_{00}$ and $\mathrm{TE}_{10}$ modes. (e) The geometry dependence of ZDW for $\mathrm{TE}_{10}$ mode. Anomalous dispersion can be ob-

tained above the ZDW. Reprinted from [225]. Distributed under a Creative Commons Attribution NonCommercial License 4.0 (CC BY-NC) http:// creativecommons.org/licenses/by-nc/4.0/.

high. Additionally, because the optical performance is dependent on the orientation of the polymer, the device has to the poled before use, and the effect of the poling on the polymer has a finite lifetime.

To overcome this barrier, researchers began investigating alternative strategies, such as dip-coating or spraycoating polymeric materials on the surfaces of integrated devices [19, 230, 231]. Because thin layers could be demonstrated, the negative effect of the polymer's loss was less, allowing higher quality factors to be achieved. Initial demonstrations focused on tailoring the thermal stability of the device, but subsequent investigations demonstrated Raman lasing behavior, albeit with very high thresholds due to the decreased quality factors because of the high loss of the polymer layer [19, 230, 231]. While successful, this fabrication approach creates devices where the polymer layer is disordered. However, molecular orientation, both with respect to the optical field and with respect to adjacent molecules, can govern the performance of the resulting device. Therefore, this strategy places fundamental limits on the types of nonlinear behaviors that can be accessed as well as the efficiencies and thresholds possible.

To realize the potential of small organic molecules in integrated photonics, fabrication methods which allowed oriented and ordered monolayers of molecules had to be developed. One such route was inspired by earlier work in the biosensor field [232-238]. By self-assembling and covalently attaching nonlinear organic molecules onto the device surface, these criteria can be achieved.

One example demonstration was based on combining a silica cavity with a monolayer of DASP molecules [232]. This strategy leveraged the intrinsic hydroxyl groups present on a silica device surface as anchor sites for the highly nonlinear DASP molecules, thus allowing all aspects of the molecular orientation to be controlled (Figure 8a). However, because the circulating optical field is primary confined within the silica device, the net effect of the molecule on the efficiency will be governed by a weighted $\chi^{(3)}$, described by the relative overlap of the optical field in each material. Despite the reduced effect, the DASP molecule is still able to significantly increase the performance (Figure $8 \mathrm{~b}$ and $\mathrm{c})$ ). In complementary work that leverages the same surface chemistry and optical cavity type, a DASP-based surface coating is used to demonstrate third harmonic generation with approximately a 4 orders of magnitude improvement in efficiency over a nonfunctionalized device [90]. This strategy allowed a nearIR laser to be used to reach the visible. Both of these examples relied on the nonlinearity present in the molecule to create a nonlinear device.

Recent work demonstrated that by perturbing the surface of a device, it is possible to improve the device's nonlinear performance. Specifically, by attaching an 


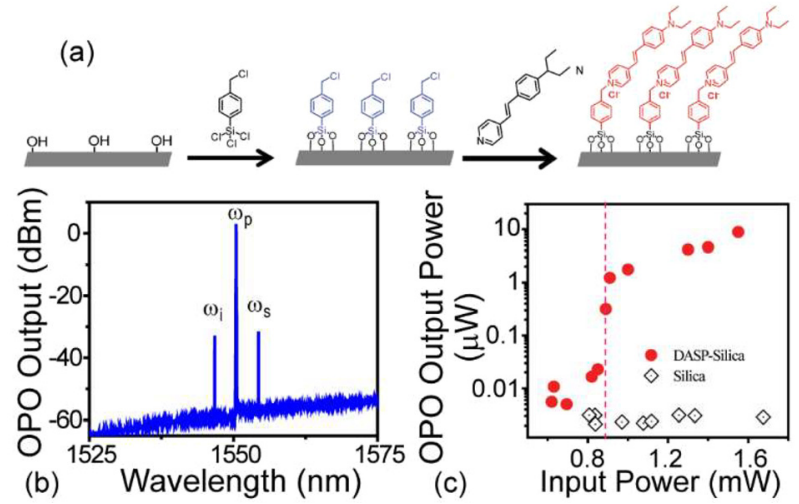

Figure 8: DASP coated silica resonant cavity for four wavemixing. (a) Overview of surface chemistry method used to create an aligned monolayer of DASP molecules on the device surface.

(b) Representative optical parametric oscillation (OPO) spectrum. (c) Threshold power comparing non-functionalized and DASP-functionalized devices. Reproduced from reference [232]. Distributed under a Creative Commons Attribution NonCommercial License 4.0 (CC BY-NC) http://creativecommons.org/licenses/by$\mathrm{nc} / 4.0 /$

oriented siloxane monolayer ( $\mathrm{Si}-\mathrm{O}-\mathrm{Si}$ ) on a silica surface, the ability of the underlying device to generate stimulated Raman scattering or create a Raman laser is enhanced due to the creation of a surface Raman mode in this oriented and ordered layer. As expected, due to the ordering, the threshold and efficiencies of this mode are polarization dependent, unlike in the amorphous device. Raman (Stokes) is a particularly flexible excitation mechanism because it is not dependent on a specific electronic transition. As such, this approach for increasing the efficiency of Stokes and Anti-Stokes generation could provide an accelerated path towards UV emission.

In the near future, it is expected that the harmonic signals can reach the UV range by precisely tuning the organic NLO materials and the frequencies/wavelengths among the pump, Raman Stokes, and parametric oscillation photons. The innovations of photonic devices are also probable as nanofabrication allows more options to design on-chip photonic devices as long as the functionalization of organic NLO materials are feasible.

\section{Future directions}

\subsection{New materials}

Recently, a new class of wide band gap semiconductor beta-phase gallium oxide $\left(\beta-\mathrm{Ga}_{2} \mathrm{O}_{3}\right)$ has emerged with many promising properties. $\beta-\mathrm{Ga}_{2} \mathrm{O}_{3}$ possesses a bandgap energy of $4.8 \mathrm{eV}$ and exhibits large laser induced damage threshold (LIDT) [239], which is promising for high power PICs. The low refractive index contrast between core and cladding layers also minimize the scattering loss [240]. Furthermore, $\beta-\mathrm{Ga}_{2} \mathrm{O}_{3}$ also has a small lattice mismatch with the InAlGaN material system, which leads to possible active integration of $\mathrm{Ga}_{2} \mathrm{O}_{3}$ photonic devices with InAlGaN lasers and detectors.

Preliminary efforts have been paid towards the fundamental optical properties as well as device performance of $\beta-\mathrm{Ga}_{2} \mathrm{O}_{3}$. H. Chen et al. characterized the optical nonlinearity of $\beta-\mathrm{Ga}_{2} \mathrm{O}_{3}$, where a TPA coefficient of $0.6-$ $3.2 \mathrm{~cm} / \mathrm{GW}$, and an $n_{2}$ in the range of $-2.1 \times 10^{-15} \mathrm{~cm}^{2} \mathrm{~W}^{-1}$ to $-2.9 \times 10^{-15} \mathrm{~cm}^{2} \mathrm{~W}^{-1}$ were obtained at $404 \mathrm{~nm}$ [128]. It's noteworthy that bulk crystalline $\beta-\mathrm{Ga}_{2} \mathrm{O}_{3}$ was obtained using the floating zone growth method [241], which leads to much superior crystal quality with less defect density on $\beta-\mathrm{Ga}_{2} \mathrm{O}_{3}$ compared to $\mathrm{GaN}$ bulk crystals. As a result, the TPA coefficient of $\beta-\mathrm{Ga}_{2} \mathrm{O}_{3}$ is lower than $\mathrm{GaN}$ (see Figures $9 \mathrm{a}$ and b). More recently, J. Zhou et al. successfully fabricated the $\beta-\mathrm{Ga}_{2} \mathrm{O}_{3}$ waveguides and analyzed their performance in the UV to near infrared (NIR) region [240] (see Figures $9 \mathrm{c}$ and d) [240]. A low propagation loss of $3.7 \mathrm{~dB} / \mathrm{cm}$ was obtained on the $\beta-\mathrm{Ga}_{2} \mathrm{O}_{3}$ waveguide at the wavelength of $810 \mathrm{~nm}$, which
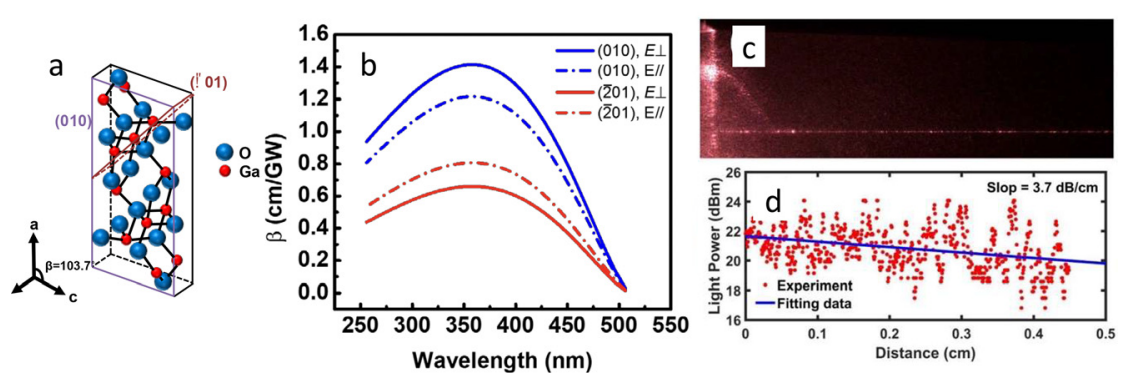

Figure 9: (a) The schematic of $\beta-\mathrm{Ga}_{2} \mathrm{O}_{3}$ crystal structures showing (010) and (2 01) crystal planes [128]. (b) The estimated wavelength dependence of TPA coefficient for (010) and (2 01) $\beta-\mathrm{Ga}_{2} \mathrm{O}_{3}$ samples. " $E \perp$ " indicates that the electrical field intensity is perpendicular to [102] direction, while " $E / /$ " indicates that field intensity is parallel to [102] direction. Reprinted with permission from [128]. Copyright 2018 by the Optical Society of America. (c) Top

image captured by the linear CMOS camera of a $\beta-\mathrm{Ga}_{2} \mathrm{O}_{3}$ waveguide with $1.5 \mu \mathrm{m}$ width at $810 \mathrm{~nm}$ wavelength. (d) Experimental data and regression analysis of $\beta-\mathrm{Ga}_{2} \mathrm{O}_{3}$ waveguide with $3.7 \mathrm{~dB} / \mathrm{cm}$ loss. Reprinted with permission from [240]. Copyright 2019 by the American Physical Society. 
is comparable to the state of the art. Combined with theoretical simulations, various loss mechanisms from twophoton absorption, sidewall scattering, top surface scattering, and bulk scattering were discussed for $\beta-\mathrm{Ga}_{2} \mathrm{O}_{3}$ waveguides, and their contributions to the total optical loss were estimated. It is expected that the performance of $\beta-\mathrm{Ga}_{2} \mathrm{O}_{3}$ photonic devices could be greatly improved with the further development in materials synthesis, optical design, and device fabrications, which will unleash the full potential of $\beta-\mathrm{Ga}_{2} \mathrm{O}_{3}$ for UV-Vis photonics applications.

In addition to emerging crystalline materials, there is a whole host of new organic materials on the horizon. One simple organic platform is Anthracene [150, 153, 242, 243]. This molecule is characterized by its unique structure which consists of three fused benzene rings, giving it a highly conjugated, resonant structure and allowing it to readily pack into a semi-crystalline structure in the condensed phase. It has previously been used as an organic dye and as a scintillator in organic semiconductors. Moreover, because of their extended conjugation length, anthracenes can make for highly nonlinear molecules due to the delocalization of $\pi$ electrons over the extent of the entire molecule [244]. This delocalization allows the molecule to become more polarized given an external oscillating field. This higher polarizability gives rise to second and third order phenomenon even at modest field strengths.

Qualitative reports on the nonlinear activity of anthracenes varies widely due to the multitude of unique derivatives which can be synthesized. Basic DFT models on anthracene crystals without additional functional groups report only modest values for $\chi^{(3)}$ [242]. In contrast, other theoretical studies report marked enhancement of the first hyperpolarizability, and therefore nonlinear optical properties, when anthracenes are coordinated with halide acids [166]. However, studies on oriented, ordered monolayers of anthracene thin films or integration of anthracenes with integrated photonic devices have yet to be performed.

If one considers moving to a slightly more complex molecular structure, experimental reports have shown large enhancements in both second-harmonic generation and third-harmonic generation when anthracene is synthesized into a highly-conjugated one-dimensional crystalline polymer, or metal organic framework (MOF) [245]. The developed MOFs ligands possess an acceptor $-\pi-$ donor- $\pi$-acceptor structure which is symmetric and a singlet biradical electronic ground state, thus boosting its $\chi^{(3)}$ and $\chi^{(5)}$ optical nonlinearities. The reported complex's $\chi^{(3)}$ can reach as high as $8 \times 10^{-11} \mathrm{esu}$, which is roughly three orders of magnitude higher than typical bulk dielectrics such as $\mathrm{SiO}_{2}$ and $\mathrm{ZnO}[246]$. When anthracene is attached along the backbone of a methyl methacrylate repeat unit in PMMA thin films, the enhancement in $\chi^{(3)}$ is even larger, reaching values of about $\sim 10^{-7}$ esu [243]. This is due to the expanded conjugation length, as the polarizable crosssection now spans the length of a polymer chain ( $\sim 100 \mathrm{kDa})$, allowing for an increased nonlinear response. Reaching $\chi^{(5)}$ optical nonlinearities in classic crystalline materials is typically not experimentally achievable due to the optical intensities that would be required. Thus, organic small molecules truly provide a route to achieve currently unavailable device performance.

Lastly, though not the focus of this work, an emerging area is creating low-power integrated optical systems by leveraging the intrinsic material properties to achieve functionality, such as phase change materials for $Q$ switching. Organic materials offer a particularly unique strategy to achieve this goal. Specifically, unlike in conventional devices where layers consist of a single material type, with organics, it is possible to attach more than one type of molecule to a device surface, creating a multifunctional multi-material monolayer. This concept was demonstrated recently using a photo-responsive group $[247,248]$, but it has yet to be extended into the realm of nonlinear optical devices.

\subsection{Inverse design to advance device performance}

Thus far, this review has focused on the relatively linear advances in new materials and in more complex device architectures. Recent advances in theoretical device design has opened the door for transformational changes.

Nanophotonic devices often span many wavelengths while having feature sizes on the order of a wavelength, implying that neither lumped nor ray optic approximations suffice and they must be modeled via full-wave solution of Maxwell's equation. The difficulty in modeling nanophotonic devices and lack of analytical solutions makes designing new devices especially challenging. Inverse design, which is the automated design of new devices given target specifications and design constraints as input via optimization, has recently emerged and seen considerable success in the nanophotonics field [249]. Although many optimization algorithms have been applied successfully towards the inverse design of nanophotonic devices, including Direct Binary Search [250] and Binary Particle Swarm Optimization [251], local gradientbased search methods have become among the most popular and successful approaches largely due to the adjoint method. 
The adjoint method, which first appeared in the context of fluid dynamics [252], enables the computation of the full gradient of the objective function being optimized with respect to the optimization variables with just two simulations regardless of the number of parameters-the forward problem and the adjoint system [253]. This is in contrast to naïve approaches such as finite difference approximations which require $\mathrm{N}+1$ simulations per gradient evaluation where $\mathrm{N}$ is the number of optimization parameters. The adjoint method has been applied to design many different devices as shown in Figure 10 including wavelength demultiplexers [254, 255], polarization splitters [250], power splitters [251], photonic switches [256], nanophotonic resonators [257], and grating couplers [258]. Furthermore, thanks to the flexibility of the gradient-based optimization approach, arbitrary nonlinear objective functions with highly complex constraints can be handled, which has enabled incorporating fabrication [259] and robustness constraints at design time [256, 260].

Although traditionally most inverse design implementations in nanophotonics have leveraged the Finite Difference Time Domain (FDTD) method [265] to solve the forward problem, recent work has shown that Boundary
Integral Equation (BIE) based techniques can be used to simulate nanophotonic devices up to several orders of magnitude faster and more accurately than finite difference and finite element approaches [261]. Furthermore, due to the discretization of global integral operators rather than local difference operators, integral equation methods are essentially dispersion-free, unlike FDTD and finite element method (FEM) which suffer significantly from numerical dispersion. Finally, since BIE methods only discretize the boundaries between different dielectric regions rather than the whole volumes, device optimization via boundary perturbation methods can be implemented naturally and seamlessly without having to resort to level-set approaches [261].

Initial research in inverse design for nanophotonics focused on passive, linear devices on Silicon-on-Insulator (SOI) platforms. Recently, the approach has been extended to time-varying active devices [266-268], topological insulators [264], and nonlinear optics [263], as well as alternative substrates such as diamond [262]. In conclusion, inverse design for nanophotonics has demonstrated a strong track record for producing high-performance, compact, and robust devices in numerous different
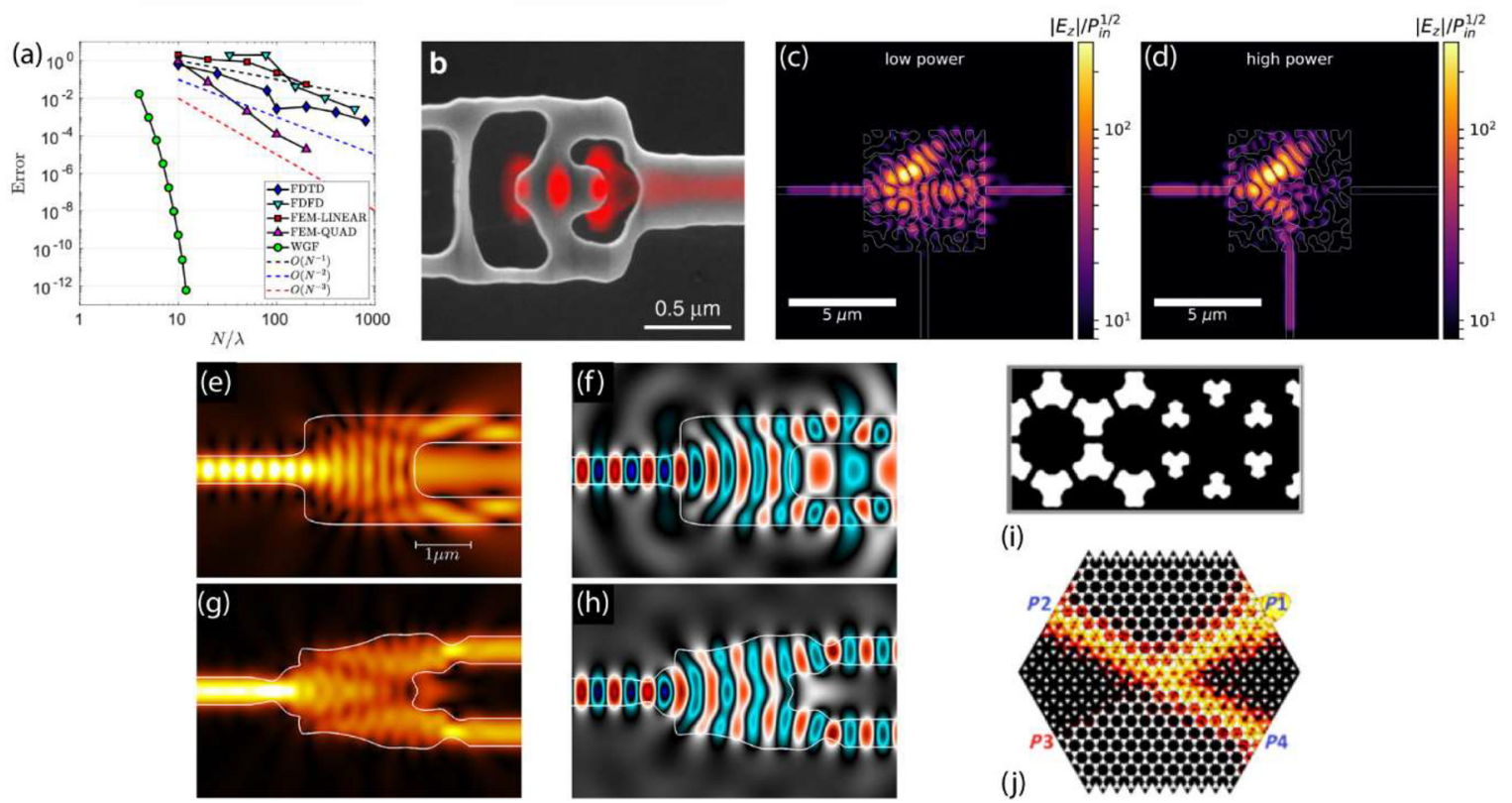

Figure 10: (a) Convergence comparison of BIE, FDTD, FDFD, and FEM solvers with respect to mesh resolution [261]. Reprinted with permission from C. Sideris, E. Garza, O. P. Bruno, ACS Photonics. 6(12):3233-40 (2019). Copyright (2019) American Chemical Society. Example devices that have been designed using inverse design include: (b) a vertical coupler on diamond substrate (Reproduced from reference [262]. Distributed under a Creative Commons Attribution NonCommercial License 4.0 (CC BY-NC) http://creativecommons.org/licenses/by-nc/4.0/), (c)/(d) nonlinear intensity-based beam splitter [263] (Reprinted with permission from T. W. Hughes, M. Minkov, I.A.D. Williamson, S. Fan, ACS Photonics. 5(12):4781-87 (2018). Copyright (2018) American Chemical Society), (e)-(h) $2 \times 2$ power splitter design using BIE solver and adjoint method before and after optimization [261] (Reprinted with permission from C. Sideris, E. Garza, O. P. Bruno, ACS Photonics. 6(12):3233-40 (2019). Copyright (2019) American Chemical Society), (i)/(j) and acoustic topological insulator [264] (Reprinted with permission from R. E. Christiansen, F. Wang, O. Sigmund, Physical Review Letters, 122 (23) (2019). Copyright (2019) by the American Physical Society). 
contexts and it has proven to be an invaluable tool for the photonics designer.

\section{Summary}

In summary, the ever-growing materials toolbox provided by emerging semiconductors and small organic molecules optimized for UV-Vis operation will continue to enable device innovation. When coupled with emerging theoretical predictive constructs, such as inverse design for accelerated device design, it is easy to anticipate rapid growth in the field. These new devices will support applications in imaging, cryptography, and spectroscopy as well as enable new fundamental science.

Acknowledgments: The authors would like to acknowledge financial support from the Air Force Office of Scientific Research (FA9550-20-1-0087), the Army Research Office (W911NF1810033, W911NF-19-1-0089, W911NF-19-1-0129), and the National Science Foundation (1849965).

Author contributions: All authors have accepted responsibility for the entire content of this manuscript and approved its submission.

Research funding: This work was supported by the Air Force Office of Scientific Research (FA9550-20-1-0087), the Army Research Office (W911NF1810033, W911NF-19-10089, W911NF-19-1-0129), and the National Science Foundation (1849965).

Competing interests: Authors state no conflict of interest.

\section{References}

[1] A. Kovach, D. Chen, J. He, et al., "Emerging material systems for integrated optical Kerr frequency combs," Adv. Opt. Photon., vol. 12, no. 1, pp. 135-222, 2020.

[2] A. L. Gaeta, M. Lipson, and T. J. Kippenberg, "Photonic-chip-based frequency combs," Nat. Photon., vol. 13, no. 3, pp. 158-169, 2019.

[3] D. Grassani, M. H. R. Pfeiffer, T. J. Kippenberg, and C.-S. Bres, "Second- and third-order nonlinear wavelength conversion in an all-optically poled Si3N4 waveguide," Opt. Lett., vol. 44, no. 1, pp. 106-109, 2019.

[4] X. Liu, A. W. Bruch, Z. Gong, et al., "Ultra-high-Q UV microring resonators based on a single-crystalline AIN platform," Optica, vol. 5, no. 10, pp. 1279-1282, 2018.

[5] A. Guarino, G. Poberaj, D. Rezzonico, R. Degl'Innocenti, and P. Guenter, "Electro-optically tunable microring resonators in lithium niobate," Nat. Photon., vol. 1, no. 7, pp. 407-410, 2007.

[6] A. Liu, R. Jones, L. Liao, et al., "A high-speed silicon optical modulator based on a metal-oxide-semiconductor capacitor," Nature, vol. 427, no. 6975, pp. 615-618, 2004.
[7] G. T. Reed, G. Mashanovich, F. Y. Gardes, and D. J. Thomson, "Silicon optical modulators," Nat. Photon., vol. 4, no. 8, pp. 518526, 2010.

[8] M. Berggren, A. Dodabalapur, Z. Bao, and R. Slusher, "Solidstate droplet laser made from an organic blend with a conjugated polymer emitter," Adv. Mater., vol. 9, no. 12, p. 968, 1997.

[9] M. Lebental, J. Lauret, R. Hierle, and J. Zyss, "Highly directional stadium-shaped polymer microlasers," Appl. Phys. Lett., vol. 88, no. 3, 2006, https://doi.org/10.1063/1.2159099.

[10] Z.-P. Liu, Y. Li, Y.-F. Xiao, B.-B. Li, X.-F. Jiang, et al., “Direct laser writing of whispering gallery microcavities by two-photon polymerization,” Appl. Phys. Lett., vol. 97, no. 21, 2010, https:// doi.org/10.1063/1.3517493.

[11] J. K. S. Poon, L. Zhu, G. A. DeRose, and A. Yariv, "Polymer microring coupled-resonator optical waveguides," IEEE J. Lightwave Technol., vol. 24, no. 4, pp. 1843-1849, 2006.

[12] A. A. Savchenkov, H. Mahalingam, V. S. Ilchenko, S. Takahashi, A. B. Matsko, et al., "Polymer waveguide couplers for fluorite microresonators," IEEE Photon. Technol. Lett., vol. 29, no. 8, pp. 667-670, 2017.

[13] A. M. Armani, A. Srinivasan, and K. J. Vahala, "Soft lithographic fabrication of high $Q$ polymer microcavity arrays," Nano Lett., vol. 7, no. 6, pp. 1823-1826, 2007.

[14] Z. Zhang, N. Yao, J. Pan, L. Zhang, W. Fang, and L. Tong, "A new route for fabricating polymer optical microcavities," Nanoscale, vol. 11, no. 12, pp. 5203-5208, 2019.

[15] T. Grossmann, S. Schleede, M. Hauser, T. Beck, M. Thiel, et al., "Direct laser writing for active and passive high- $Q$ polymer microdisks on silicon," Opt. Express, vol. 19, no. 12, pp. 1145111456, 2011.

[16] D. Rezzonico, M. Jazbinsek, A. Guarino, O.-P. Kwon, and P. Günter, "Electro-optic Charon polymeric microring modulators," Opt. Express, OE., vol. 16, no. 2, pp. 613-627, 2008.

[17] P. Del'Haye, A. Schliesser, O. Arcizet, T. Wilken, R. Holzwarth, and T. J. Kippenberg, "Optical frequency comb generation from a monolithic microresonator," Nature, vol. 450, no. 7173, pp. 1214-1217, 2007, https://doi.org/10.1038/nature06401.

[18] R. Castro-Beltrán, V. M. Diep, S. Soltani, E. Gungor, and A. M. Armani, "Plasmonically enhanced Kerr frequency combs," ACS Photon., vol. 4, no. 11, pp. 2828-2834, 2017.

[19] B.-B. Li, Y.-F. Xiao, M.-Y. Yan, W. R. Clements, and Q. Gong, "Low-threshold Raman laser from an on-chip, high-Q, polymercoated microcavity," Opt. Lett., vol. 38, no. 11, pp. 1802-1804, 2013.

[20] N. Deka, A. J. Maker, and A. M. Armani, "Titanium-enhanced Raman microcavity laser," Opt. Lett., vol. 39, no. 6, p. 1354, 2014.

[21] S. Fujii, T. Kato, R. Suzuki, A. Hori, and T. Tanabe, "Transition between Kerr comb and stimulated Raman comb in a silica whispering gallery mode microcavity,"J. Opt. Soc. Am. B, JOSAB., vol. 35, no. 1, pp. 100-106, 2018.

[22] D. Martyshkin, V. Fedorov, T. Kesterson, et al., "Visible-nearmiddle infrared spanning supercontinuum generation in a silicon nitride $\left(\mathrm{Si}_{3} \mathrm{~N}_{4}\right)$ waveguide," Opt. Mater. Express, vol. 9, no. 6, pp. 2553-2559, 2019.

[23] R. Holzwarth, M. Zimmermann, T. Udem, and T. Hansch, “Optical clockworks and the measurement of laser frequencies with a mode-locked frequency comb," IEEE J. Quantum Electron., vol. 37, no. 12, pp. 1493-1501, 2001. 
[24] S. B. Papp, K. Beha, P. Del'Haye, et al., “Microresonator frequency comb optical clock," Optica, vol. 1, no. 1, pp. 10-14, 2014.

[25] Z. L. Newman, V. Maurice, T. Drake, et al., "Architecture for the photonic integration of an optical atomic clock," Optica, vol. 6, no. 5, pp. 680-685, 2019.

[26] J. Kitching, “Chip-scale atomic devices," Appl. Phys. Rev., vol. 5, no. 3, 2018, https://doi.org/10.1063/1.5026238.

[27] H. Utzat, W. Sun, A. E. K. Kaplan, et al., "Coherent single-photon emission from colloidal lead halide perovskite quantum dots," Science, vol. 363, no. 6431, p. 1068+, 2019.

[28] W. Zipfel, R. Williams, and W. Webb, "Nonlinear magic: multiphoton microscopy in the biosciences," Nat. Biotechnol., vol. 21, no. 11, pp. 1368-1376, 2003.

[29] V. V. Tuchin, "Polarized light interaction with tissues," J. Biomed. Opt., vol. 21, no. 7, 2016, https://doi.org/10.1117/1.jbo.21.7. 071114.

[30] F. Wang, H. Wan, Z. Ma, et al., "Light-sheet microscopy in the near-infrared II window," Nat. Methods, vol. 16, no. 6, p. 545+, 2019.

[31] D. Larson, W. Zipfel, R. Williams, et al., "Water-soluble quantum dots for multiphoton fluorescence imaging in vivo," Science, vol. 300, no. 5624, pp. 1434-1436, 2003.

[32] E. Hemmer, P. Acosta-Mora, J. Mendez-Ramos, and S. Fischer, “Optical nanoprobes for biomedical applications: shining a light on upconverting and near-infrared emitting nanoparticles for imaging, thermal sensing, and photodynamic therapy," J. Mater. Chem. B, vol. 5, no. 23, pp. 4365-4392, 2017.

[33] B. Weigelin, G.-J. Bakker, and P. Friedl, "Third harmonic generation microscopy of cells and tissue organization," J. Cell. Sci., vol. 129, no. 2, pp. 245-255, 2016.

[34] P. Huber, "High power in the near ultraviolet using efficient SHG," Opt. Commun., vol. 15, no. 2, pp. 196-200, 1975.

[35] Z. Chen, D. Cui, M. Li, C. Jiang, J. Zhou, and G. Yang, “High conversion efficiency of 2 nd harmonic generation in step quantum-wells," Appl. Phys. Lett., vol. 61, no. 20, pp. 24012402, 1992.

[36] Z. Wang, B. Teng, K. Fu, et al., "Efficient second harmonic generation of pulsed laser radiation in BiB306 (BIBO) crystal with different phase matching directions," Opt. Commun., vol. 202, no. 1, pp. 217-220, 2002.

[37] M. A.Rahman, and I.Alghoraibi, "Theoretical investigation of second harmonic efficiency effect on third harmonic conversion efficiency in BBO crystals,". Optik vol. 194, 2019. https://doi. org/10.1016/j.ijleo.2019.163031.

[38] P. Abolghasem, J. Han, B. J. Bijlani, and A. S. Helmy, “Type0 second order nonlinear interaction in monolithic waveguides of isotropic semiconductors," Opt. Express, vol. 18, no. 12, pp. 12681-12689, 2010.

[39] R. Luo, Y. He, H. Liang, M. Li, and Q. Lin, "Highly tunable efficient second-harmonic generation in a lithium niobate nanophotonic waveguide," Optica, vol. 5, no. 8, p. 1006, 2018.

[40] C. Wang, C. Langrock, A. Marandi, et al., "Ultrahigh-efficiency wavelength conversion in nanophotonic periodically poled lithium niobate waveguides," Optica, vol. 5, no. 11, p. 1438, 2018.

[41] D. Coquillat, G. Vecchi, C. Comaschi, A. Malvezzi, J. Torres, and M. d'Yerville, "Enhanced second- and third-harmonic generation and induced photoluminescence in a two-dimensional GaN photonic crystal," Appl. Phys. Lett., vol. 87, no. 10, 2005, https:// doi.org/10.1063/1.2037849.
[42] L. Gao, "Second- and third-harmonic generations in compositionally graded films," Phys. Rev. E, vol. 71, no. 6, pp. 2, 2005.

[43] C. Huang, and Y. Zhu, "Third harmonic generation in a periodic structure with simultaneous linear and nonlinear modulation," Phys. Status Solidi B-Basic Solid State Phys., vol. 242, no. 8, pp. 1694-1699, 2005.

[44] X. Liu, D. Li, P. Shi, et al., "Highly efficient third-harmonic generation with electro-optically Q-switched diode-end-purnped Nd : YVO4 slab laser," Opt. Commun., vol. 272, no. 1, pp. 192196, 2007.

[45] H. Kitano, T. Matsui, K. Sato, et al., "Efficient 355-nm generation in CsB305 crystal,” Opt. Lett., vol. 28, no. 4, pp. 263-265, 2003.

[46] M. A. Knyazev and S. A. Kozlov, "Control of the third-harmonic generation efficiency upon interaction of few-cycle waves in nonlinear media," Quantum Electron., vol. 48, no. 2, pp. 119123, 2018.

[47] A. A. Savchenkov, A. B. Matsko, V. S. Ilchenko, I. Solomatine, D. Seidel, and L. Maleki, "Tunable optical frequency comb with a crystalline whispering gallery mode resonator," Phys. Rev. Lett., vol. 101, no. 9, 2008, https://doi.org/10.1103/physrevlett.101. 093902.

[48] Y. K. Chembo, D. V. Strekalov, and N. Yu, "Spectrum and dynamics of optical frequency combs generated with monolithic whispering gallery mode resonators," Phys. Rev. Lett., vol. 104, no. 10, 2010, https://doi.org/10.1103/physrevlett.104.103902.

[49] Y. Okawachi, K. Saha, J. S. Levy, Y. H. Wen, M. Lipson, and A. L. Gaeta, "Octave-spanning frequency comb generation in a silicon nitride chip,” Opt. Lett., vol. 36, no. 17, pp. 3398-3400, 2011.

[50] S. B. Papp, P. Del'Haye, and S. A. Diddams, "Mechanical control of a microrod-resonator optical frequency comb," Phys. Rev. X, vol. 3, no. 3, 2013, https://doi.org/10.1103/physrevx.3.031003.

[51] A. G. Griffith, R. K. W. Lau, J. Cardenas, et al., "Silicon-chip midinfrared frequency comb generation," Nat. Commun., vol. 6, 2015, https://doi.org/10.1038/ncomms7299.

[52] Y. Nakagawa, Y. Mizumoto, T. Kato, et al., “Dispersion tailoring of a crystalline whispering gallery mode microcavity for a widespanning optical Kerr frequency comb," J. Opt. Soc. Am. B, JOSAB., vol. 33, no. 9, pp. 1913-1920, 2016.

[53] X. Xue, X. Zheng, B. Zhou, and A. M. Weiner, "Microresonator frequency combs for integrated microwave photonics," IEEE Photon. Technol. Lett., vol. 30, no. 21, pp. 1814-1817, 2018.

[54] M. Kues, C. Reimer, J. M. Lukens, et al., "Quantum optical microcombs," Nat. Photon., vol. 13, no. 3, pp. 170-179, 2019.

[55] D. Chen, A. Kovach, S. Poust, V. Gambin, and A. M. Armani, "Normal dispersion silicon oxynitride microresonator Kerr frequency combs," Appl. Phys. Lett., vol. 115, no. 5, p. 1105, 2019.

[56] K. E. Webb, Y. Q. Xu, M. Erkintalo, and S. G. Murdoch, "Generalized dispersive wave emission in nonlinear fiber optics," Opt. Lett., vol. 38, no. 2, p. 151, 2013.

[57] M. Fejer, G. Magel, D. Jundt, and R. Byer, "Quasi-phase-matched 2nd harmonic-generation - tuning and tolerances," IEEE J. Quantum Electron., vol. 28, no. 11, pp. 2631-2654, 1992.

[58] J. S. Levy, M. A. Foster, A. L. Gaeta, and M. Lipson, "Harmonic generation in silicon nitride ring resonators," Opt. Express, vol. 19, no. 12, pp. 11415-11421, 2011.

[59] Y. Li, S. H. Wang, Y. Tian, et al., "Third-harmonic generation in CMOS-compatible highly doped silica micro-ring resonator," Opt. Express, vol. 28, no. 1, pp. 641-651, 2020. 
[60] Y. J. Ding, "Investigation of anti-Stokes Raman processes at phonon-polariton resonance: from Raman oscillation, frequency upconversion to Raman amplification," Opt. Lett., vol. 40, no. 5, pp. 729-732, 2015.

[61] J. Moore, M. Tomes, T. Carmon, and M. Jarrahi, “Continuouswave cascaded-harmonic generation and multi-photon Raman lasing in lithium niobate whispering-gallery resonators," Appl. Phys. Lett., vol. 99, no. 22, 2011, https://doi.org/10.1063/1. 3665947.

[62] B. Feigel, H. Thienpont, and N. Vermeulen, "Design of infrared and ultraviolet Raman lasers based on grating-coupled integrated diamond ring resonators," J. Opt. Soc. Am. B - Opt. Phys., vol. 33, no. 3, pp. B5-B18, 2016.

[63] M. Kauranen, and A. V. Zayats, "Nonlinear plasmonics," Nat. Photon., vol. 6, no. 11, pp. 737-748, 2012.

[64] F. Peyskens, P. Wuytens, A. Raza, P. Van Dorpe, and R. Baets, "Waveguide excitation and collection of surface-enhanced Raman scattering from a single plasmonic antenna," Nanophotonics, vol. 7, no. 7, pp. 1299-1306, 2018.

[65] B. J. Eggleton, C. G. Poulton, P. T. Rakich, M. J. Steel, and G. Bahl, "Brillouin integrated photonics," Nat. Photonics., vol. 13, no. 10, pp. 664-677, 2019.

[66] J. P. Epping, T. Hellwig, M. Hoekman, et al., "On-chip visible-toinfrared supercontinuum generation with more than $495 \mathrm{THz}$ spectral bandwidth," Opt. Express, vol. 23, no. 15, p. 19596, 2015.

[67] B. Xiang, X. Ren, S. Ruan, et al., "Visible to near-infrared supercontinuum generation in yttrium orthosilicate bulk crystal and ion implanted planar waveguide," Sci Rep, vol. 6, no. 1, p. 31612, 2016

[68] P. Innocenzi, "Infrared spectroscopy of sol-gel derived silicabased films: a spectra-microstructure overview," J. Non-Cryst. Solids, vol. 316, nos 2-3, pp. 309-319, 2003.

[69] H. Shang, Y. Wang, S. Limmer, T. Chou, K. Takahashi, and G. Cao, "Optically transparent superhydrophobic silica-based films," Thin Solid Films, vol. 472, nos 1-2, pp. 37-43, 2005.

[70] R. Ciriminna, A. Fidalgo, V. Pandarus, F. Beland, L. M. Ilharco, and M. Pagliaro, "The sol-gel route to advanced silica-based materials and recent applications," Chem. Rev., vol. 113, no. 8, pp. 6592-6620, 2013.

[71] J. L. Gurav , I.-K. Jung, H.-H. Park, E. S. Kang, D. Y. Nadargi “Silica aerogel: synthesis and applications,". J. Nanomater. vol. 2010, Art no. 409310, 2010 https://doi.org/10.1155/2010/409310.

[72] J. Endo, A. Ito, T. Kimura, and T. Goto, "High-speed deposition of dense, dendritic and porous $\mathrm{SiO} 2$ films by $\mathrm{Nd}$ : YAG laser chemical vapor deposition," Mater. Sci. Eng. B -Adv. Func. SolidState Mater., vol. 166, no. 3, pp. 225-229, 2010.

[73] T. loppolo and M. V. Ötügen, "Pressure tuning of whispering gallery mode resonators," J. Opt. Soc. Am. B, JOSAB., vol. 24, no. 10, pp. 2721-2726, 2007.

[74] Y.-S. Park and H. Wang, "Resolved-sideband and cryogenic cooling of an optomechanical resonator," Nat. Phys., vol. 5, no. 7, pp. 489-493, 2009.

[75] M. Pöllinger and A. Rauschenbeutel, “All-optical signal processing at ultra-low powers in bottle microresonators using the Kerr effect," Opt. Express, vol. 18, no. 17, pp. 17764-17775, 2010.

[76] X. Zhang and A. M. Armani, "Suspended bridge-like silica $2 \times 2$ beam splitter on silicon,” Opt. Lett., vol. 36, no. 15, p. 3012, 2011
[77] A. J. Maker and A. M. Armani, "Nanowatt threshold, alumina sensitized neodymium laser integrated on silicon," Opt. Express, vol. 21, no. 22, p. 27238, 2013.

[78] X. Zhang and A. M. Armani, "Silica microtoroid resonator sensor with monolithically integrated waveguides," Opt. Express, vol. 21, no. 20, p. 23592, 2013.

[79] W. Yoshiki and T. Tanabe, "All-optical switching using Kerr effect in a silica toroid microcavity," Opt. Express, vol. 22, no. 20, pp. 24332-24341, 2014

[80] J. Kim, M. C. Kuzyk, K. Han, H. Wang, and G. Bahl, "Nonreciprocal Brillouin scattering induced transparency," Nat. Phys., vol. 11, no. 3, pp. 275-280, 2015.

[81] C. Milian, A. V. Gorbach, M. Taki, A. V. Yulin, and D. V. Skryabin, "Solitons and frequency combs in silica microring resonators: interplay of the Raman and higher-order dispersion effects," Phys. Rev. A, vol. 92, no. 3, 2015, https://doi.org/10.1103/ physreva.92.033851.

[82] R. Myers, N. Mukherjee, and S. Bruek, "Large 2nd-order nonlinearity in poled fused-silica," Opt. Lett., vol. 16, no. 22, pp. 1732-1734, 1991.

[83] V. Ilchenko and A. Matsko, "Optical resonators with whisperinggallery modes - Part II: Applications," IEEE J. Sel. Top. Quantum Electron., vol. 12, no. 1, pp. 15-32, 2006.

[84] Y. Li, X. Jiang, G. Zhao, and L. Yang, "Whispering gallery mode microresonator for nonlinear optics,".arXiv:1809.04878 [physics], 2018

[85] G. Lin, A. Coillet, and Y. K. Chembo, "Nonlinear photonics with high-Q whispering-gallery-mode resonators," Adv. Opt. Photon., vol. 9, no. 4, pp. 828-890, 2017.

[86] T. Kippenberg, S. Spillane, and K. Vahala, "Kerr-nonlinearity optical parametric oscillation in an ultrahigh-Q toroid microcavity," Phys. Rev. Lett., vol. 93, no. 8, 2004, https://doi. org/10.1103/physrevlett.93.083904.

[87] A. Schliesser, G. Anetsberger, R. Rivière, O. Arcizet, and T. J. Kippenberg, "High-sensitivity monitoring of micromechanical vibration using optical whispering gallery mode resonators," New J. Phys., vol. 10, no. 9, 2008, Art no. 095015.

[88] T. J. Kippenberg, R. Holzwarth, and S. A. Diddams, "Microresonator-based optical frequency combs," Science, vol. 332, no. 6029, pp. 555-559, 2011.

[89] X. Jiang and L. Yang, "Optothermal dynamics in whisperinggallery microresonators," Light: Sci. Appl., vol. 9, no. 1, p. 24, 2020.

[90] J. Chen, X. Shen, S.-J. Tang, Q.-T. Cao, Q. Gong, and Y.-F. Xiao, "Microcavity nonlinear optics with an organically functionalized surface," Phys. Rev. Lett., vol. 123, no. 17, p. 173902, 2019.

[91] S. Spillane, T. Kippenberg, and K. Vahala, "Ultralow-threshold Raman laser using a spherical dielectric microcavity," Nature, vol. 415, no. 6872, pp. 621-623, 2002.

[92] M. V. Chistiakova, and A. M. Armani, "Cascaded Raman microlaser in air and buffer," Opt. Lett., vol. 37, no. 19, p. 4068, 2012.

[93] G. Zhao, Ş. K. Özdemir, T. Wang, et al., "Raman lasing and Fano lineshapes in a packaged fiber-coupled whispering-gallerymode microresonator," Sci. Bull., vol. 62, no. 12, pp. 875-878, 2017.

[94] B. Dunn and J. Zink, "Optical-properties of sol-gel glasses doped with organic-molecules," J. Mater. Chem., vol. 1, no. 6, pp. 903-913, 1991. 
[95] K. Binnemans, "Lanthanide-based luminescent hybrid materials," Chem. Rev., vol. 109, no. 9, pp. 4283-4374, 2009.

[96] H.-S. Hsu, C. Cai, and A. M. Armani, "Ultra-low-threshold Er:Yb sol-gel microlaser on silicon," Opt. Express, vol. 17, no. 25, pp. 23265-23271, 2009.

[97] C. Shi, S. Soltani, and A. M. Armani, “Gold nanorod plasmonic upconversion microlaser," Nano Lett., vol. 13, no. 12, pp. 58275831, 2013.

[98] H. Choi and A. M. Armani, "High efficiency Raman lasers based on Zr-doped silica hybrid microcavities," ACS Photon., vol. 3, no. 12, pp. 2383-2388, 2016.

[99] H. Choi and A. M. Armani, "Raman-Kerr frequency combs in Zrdoped silica hybrid microresonators," Opt. Lett., vol. 43, no. 12, p. 2949, 2018.

[100] A. Z. Subramanian, P. Neutens, A. Dhakal, et al., "Low-loss singlemode PECVD silicon nitride photonic wire waveguides for 532-900 nm wavelength window fabricated within a CMOS pilot line," IEEE Photon. J, vol. 5, no. 6, 2013, https://doi.org/10. 1109/jphot.2013.2292698.

[101] M. W. Puckett, R. Sharma, H.-H. Lin, M.-H. Yang, F. Vallini, and Y. Fainman, "Observation of second-harmonic generation in silicon nitride waveguides through bulk nonlinearities," Opt. Express, vol. 24, no. 15, pp. 16923-16933, 2016.

[102] K. Ikeda, R. E. Saperstein, N. Alic, and Y. Fainman, "Thermal and Kerr nonlinear properties of plasma-deposited silicon nitride/ silicon dioxide waveguides," Opt. Express, vol. 16, no. 17, pp. 12987-12994, 2008.

[103] C. J. Kruckel, A. Fulop, T. Klintberg, J. Bengtsson, P. A. Andrekson, and V. Torres-Company, "Linear and nonlinear characterization of low-stress high-confinement silicon-rich nitride waveguides," Opt. Express, vol. 23, no. 20, pp. $25827-$ 25837, 2015.

[104] J. S. Levy, A. Gondarenko, M. A. Foster, A. C. Turner-Foster, A. L. Gaeta, and M. Lipson, "CMOS-compatible multiple-wavelength oscillator for on-chip optical interconnects," Nat. Photon., vol. 4, no. 1, pp. 37-40, 2010.

[105] D. J. Moss, R. Morandotti, A. L. Gaeta, and M. Lipson, “New CMOS-compatible platforms based on silicon nitride and Hydex for nonlinear optics," Nat. Photon., vol. 7, no. 8, pp. 597-607, 2013.

[106] W. D. Sacher, Y. Huang, G.-Q. Lo, and J. K. S. Poon, "Multilayer silicon nitride-on-silicon integrated photonic platforms and devices," J. Lightwave Technol., vol. 33, no. 4, pp. 901-910, 2015.

[107] M. Prabhu, C. Roques-Carmes, Y. Shen, et al., "Accelerating recurrent sing machines in photonic integrated circuits," Optica, vol. 7, no. 5, pp. 551-558, 2020.

[108] R. Schiek and T. Pertsch, "Absolute measurement of the quadratic nonlinear susceptibility of lithium niobate in waveguides," Opt. Mater. Express, vol. 2, no. 2, pp. 126-139, 2012.

[109] C. Wang, M. Zhang, X. Chen, et al., "Integrated lithium niobate electro-optic modulators operating at CMOS-compatible voltages," Nature, vol. 562, no. 7725, p. 101+, 2018.

[110] G. Poberaj, H. Hu, W. Sohler, and P. Guenter, "Lithium niobate on insulator (LNOI) for micro-photonic devices," Laser Photon. Rev., vol. 6, no. 4, pp. 488-503, 2012.

[111] B. Desiatov, A. Shams-Ansari, M. Zhang, C. Wang, and M. Loncar, "Ultra-low-loss integrated visible photonics using thinfilm lithium niobate," Optica, vol. 6, no. 3, pp. 380-384, 2019.
[112] D. M. Lukin, C. Dory, M. A. Guidry, et al., "4H-silicon-carbide-oninsulator for integrated quantum and nonlinear photonics," Nat. Photon., vol. 14, no. 5, pp. 330-334, 2020.

[113] H. Sato, M. Abe, I. Shoji, J. Suda, and T. Kondo, "Accurate measurements of second-order nonlinear optical coefficients of $6 \mathrm{H}$ and $4 \mathrm{H}$ silicon carbide," J. Opt. Soc. Am. B., vol. 26, no. 10, pp. 1892-1896, 2009.

[114] M. W. Doherty, N. B. Manson, P. Delaney, F. Jelezko, J. Wrachtrup, and L. C. L. Hollenberg, "The nitrogen-vacancy colour centre in diamond," Phys. Rep., vol. 528, no. 1, pp. 1-45, 2013.

[115] B. J. M. Hausmann, I. Bulu, V. Venkataraman, P. Deotare, and M. Loncar, "Diamond nonlinear photonics," Nat. Photon., vol. 8, no. 5, pp. 369-374, 2014.

[116] G. N. West, W. Loh, D. Kharas, et al., "Low-loss integrated photonics for the blue and ultraviolet regime," APL Photon., vol. 4, no. 2, 2019, https://doi.org/10.1063/1.5052502.

[117] A. Major, F. Yoshino, I. Nikolakakos, J. Aitchison, and P. Smith, "Dispersion of the nonlinear refractive index in sapphire," Opt. Lett., vol. 29, no. 6, pp. 602-604, 2004.

[118] L. Chang, W. Xie, H. Shu, et al., "Ultra-efficient frequency comb generation in AlGaAs-on-insulator microresonators," Nat. Commun., vol. 11, no. 1, p. 1331, 2020.

[119] S. P. DenBaars, D. Feezell, K. Kelchner, et al., “Development of gallium-nitride-based light-emitting diodes (LEDs) and laser diodes for energy-efficient lighting and displays," ACTA Mater., vol. 61, no. 3, pp. 945-951, 2013.

[120] M. Hua, C. Liu, S. Yang, et al., "GaN-based metal-insulatorsemiconductor high-electron-mobility transistors using lowpressure chemical vapor deposition SiNx as gate dielectric," IEEE Electron Device Lett., vol. 36, no. 5, pp. 448-450, 2015.

[121] M. Abe, H. Sato, I. Shoji, et al., "Accurate measurement of quadratic nonlinear-optical coefficients of gallium nitride," J. Opt. Soc. Am. B - Opt. Phys., vol. 27, no. 10, pp. 2026-2034, 2010.

[122] C. Sun, J. Liang, J. Wang, et al., "Two-photon absorption study of GaN,” Appl. Phys. Lett., vol. 76, no. 4, pp. 439-441, 2000.

[123] H. Chen, X. Huang, H. Fu, et al., "Characterizations of nonlinear optical properties on GaN crystals in polar, nonpolar, and semipolar orientations," Appl. Phys. Lett., vol. 110, no. 18, 2017, https://doi.org/10.1063/1.4983026.

[124] Y. Fang, F. Zhou, J. Yang, et al., "Anisotropy of two-photon absorption and free-carrier effect in nonpolar GaN," Appl. Phys. Lett., vol. 106, no. 13, 2015, https://doi.org/10.1063/1. 4916829.

[125] Y. Toda, T. Matsubara, R. Morita, et al., "Two-photon absorption and multiphoton-induced photoluminescence of bulk GaN excited below the middle of the band gap," Appl. Phys. Lett., vol. 82, no. 26, pp. 4714-4716, 2003.

[126] P. Scajev, K. Jarasiunas, S. Okur, U. Oezguer, and H. Morkoc, “Carrier dynamics in bulk GaN," J. Appl. Phys., vol. 111, no. 2, 2012, https://doi.org/10.1063/1.3673851.

[127] Y. Fang, Z. Xiao, X. Wu, et al., "Optical nonlinearities and ultrafast all-optical switching of m-plane $\mathrm{GaN}$ in the nearinfrared," Appl. Phys. Lett., vol. 106, no. 25, 2015, https://doi. org/10.1063/1.4923184.

[128] H. Chen, H. Fu, X. Huang, et al., "Characterizations of the nonlinear optical properties for (010) and ((2)over-bar01) betaphase gallium oxide," Opt. Express, vol. 26, no. 4, pp. 3938-3946, 2018. 
[129] A. Majkic, A. Franke, R. Kirste, et al., "Optical nonlinear and electro-optical coefficients in bulk aluminium nitride single crystals," Phys. Status Solidi B-Basic Solid State Phys., vol. 254, no. 9, 2017, https://doi.org/10.1002/pssb.201700077.

[130] X. Guo, C.-L. Zou, and H. X. Tang, "Second-harmonic generation in aluminum nitride microrings with $2500 \%$ /W conversion efficiency," Optica, vol. 3, no. 10, pp. 1126-1131, 2016, https:// doi.org/10.1364/OPTICA.3.001126.

[131] X. Guo, C.-L. Zou, H. Jung, and H. X. Tang, "On-chip strong coupling and efficient frequency conversion between telecom and visible optical modes," Phys. Rev. Lett., vol. 117, no. 12, 2016, https://doi.org/10.1103/physrevlett.117.123902.

[132] M. Zhao, C.-H. Xu, W.-J. Hu, W.-J. Wang, L.-W. Guo, and X.-L. Chen, "Observation of two-photon absorption and nonlinear refraction in AIN," Chinese Phy. Lett., vol. 33, no. 10, 2016, https://doi.org/10.1088/0256-307x/33/10/104201.

[133] H. Jung, C. Xiong, K. Y. Fong, X. Zhang, and H. X. Tang, "Optical frequency comb generation from aluminum nitride microring resonator," Opt. Lett., vol. 38, no. 15, pp. 28102813, 2013.

[134] M. Sheik-Bahae, D. C. Hutchings, D. J. Hagan, and E. W. Van Stryland, "Dispersion of bound electron nonlinear refraction in solids," IEEE J. Quantum Electron., vol. 27, no. 6, pp. 1296-1309, 1991.

[135] J. Cimek, N. Liaros, S. Couris, R. Stepien, M. Klimczak, and R. Buczynski, "Experimental investigation of the nonlinear refractive index of various soft glasses dedicated for development of nonlinear photonic crystal fibers," Opt. Mater. Express, vol. 7, no. 10, pp. 3471-3483, 2017.

[136] A. J. Maker and A. M. Armani, "Low-loss silica-on-silicon waveguides,” Opt. Lett., vol. 36, no. 19, p. 3729, 2011.

[137] C. Xu, T. Yan, G. Wang, W. Wang, J. Liang, and X. Chen, "Spectral broadening induced by intense ultra-short pulse in $4 \mathrm{H}-\mathrm{SiC}$ crystals," Chinese Phys. B, vol. 25, no. 6, 2016, https://doi.org/ 10.1088/1674-1056/25/6/064206.

[138] G. Pandraud, H. T. M. Pham, P. J. French, and P. M. Sarro, "PECVD SiC optical waveguide loss and mode characteristics," Opt. Laser Technol., vol. 39, no. 3, pp. 532-536, 2007.

[139] K. A. Rutkowska, D. Duchesne, M. Volatier, R. Ares, V. Aimez, and R. Morandotti, "Second harmonic generation in AlGaAs nanowaveguides," Acta Phys. Pol. A, vol. 120, no. 4, pp. 725731, 2011.

[140] X. Guan, H. Hu, L. K. Oxenlowe, and L. H. Frandsen, “Compact titanium dioxide waveguides with high nonlinearity at telecommunication wavelengths," Opt. Express, vol. 26, no. 2, pp. 1055-1063, 2018.

[141] J. T. Choy, J. D. B. Bradley, P. B. Deotare, et al., "Integrated TiO2 resonators for visible photonics," Opt. Lett., vol. 37, no. 4, pp. 539-541, 2012.

[142] H. Li, F. Zhou, X. Zhang, and W. Ji, "Picosecond Z-scan study of bound electronic Kerr effect in LiNbO3 crystal associated with two-photon absorption," Appl. Phys. B, vol. 64, no. 6, pp. 659662, 1997.

[143] H. Chen, H. Fu, X. Huang, et al., "Low loss GaN waveguides at the visible spectral wavelengths for integrated photonics applications," Opt. Express, vol. 25, no. 25, pp. 31758-31773, 2017.

[144] H. Jung and H. X. Tang, "Aluminum nitride as nonlinear optical material for on-chip frequency comb generation and frequency conversion," Nanophotonics, vol. 5, no. 2, pp. 263271, 2016.

[145] L. Dalton "Nonlinear optical polymeric materials: from chromophore design to commercial applications,". Polym. Photon. Appl. I, vol. 158, pp. 1-86 2002. https://doi.org/10. 1007/3-540-44608-7_1.

[146] A. Facchetti, "Pi-conjugated polymers for organic electronics and photovoltaic cell applications," Chem. Mater., vol. 23, no. 3, pp. 733-758, 2011.

[147] L. Dalton, W. Steier, B. Robinson, et al., "From molecules to opto-chips: organic electro-optic materials," J. Mater. Chem., vol. 9, no. 9, pp. 1905-1920, 1999.

[148] A. Kulkarni, C. Tonzola, A. Babel, and S. Jenekhe, "Electron transport materials for organic light-emitting diodes," Chem. Mater., vol. 16, no. 23, pp. 4556-4573, 2004.

[149] R. Medishetty, J. K. Zareba, D. Mayer, M. Samoc, and R. A. Fischer, "Nonlinear optical properties, upconversion and lasing in metal-organic frameworks," Chem. Soc. Rev., vol. 46, no. 16, pp. 4976-5004, 2017.

[150] P. Taylor, A. Wylie, J. Huuskonen, and H. Anderson, "Enhanced electronic conjugation in anthracene-linked porphyrins," Angew. Chem. Int. Ed., vol. 37, no. 7, pp. 986-989, 1998.

[151] A. Winter, and U. S. Schubert, "Synthesis and characterization of metallo-supramolecular polymers," Chem. Soc. Rev., vol. 45, no. 19, pp. 5311-5357, 2016.

[152] Z. Yuan, N. Taylor, R. Ramachandran, and T. Marder, "Thirdorder nonlinear optical properties of organoboron compounds: molecular structures and second hyperpolarizabilities," Appl. Organomet. Chem., vol. 10, no. 3-4, pp. 305-316, 1996.

[153] W. Yang, C. Kim, M. Jeong, et al., "Synthesis and two-photon absorption properties of 9,10-bis(arylethynyl)anthracene derivatives," Chem. Mater., vol. 16, no. 14, pp. 2783-2789, 2004.

[154] J. C. S. Costa, R. J. S. Taveira, C. F. R. A. C. Lima, A. Mendes, and L. Santos, "Optical band gaps of organic semiconductor materials," Opt. Mater., vol. 58, pp. 51-60, 2016.

[155] T. He, Y. Wang, X. Tian, et al., "An organic dye with very large Stokes-shift and broad tunability of fluorescence: potential two-photon probe for bioimaging and ultra-sensitive solidstate gas sensor," Appl. Phys. Lett., vol. 108, no. 1, 2016, https://doi.org/10.1063/1.4939232.

[156] D. L. Elder, C. Haffner, W. Heni, et al., "Effect of rigid bridgeprotection units, quadrupolar interactions, and blending in organic electro-optic chromophores," Chem. Mater., vol. 29, no. 15, pp. 6457-6471, 2017.

[157] G. W. Ejuh, N. Samuel, T. N. Fridolin, and N. J. Marie, "Computational determination of the electronic and nonlinear optical properties of the molecules 2-(4-aminophenyl) quinoline, 4-(4-aminophenyl) quinoline, anthracene, anthraquinone and phenanthrene," Mater. Lett., vol. 178, pp. 221-226, 2016.

[158] T. Marks and M. Ratner, "Design, synthesis, and properties of molecule-based assemblies with large 2nd-order optical nonlinearities," Angew. Chem. Int. Ed., vol. 34, no. 2, pp. 155173, 1995.

[159] T. Verbiest, S. Houbrechts, M. Kauranen, K. Clays, and A. Persoons, "Second-order nonlinear optical materials: recent advances in chromophore design," J. Mater. Chem., vol. 7, no. 11, pp. 2175-2189, 1997.

[160] R. W. Boyd, Nonlinear Optics, Academic Press, 2008. 
[161] S. Bruni, E. Cariati, F. Cariati, F. A. Porta, S. Quici, and D. Roberto, "Determination of the quadratic hyperpolarizability of trans-4-[4-(dimethylamino)styryl]pyridine and 5-dimethylamino-1,10-phenanthroline from solvatochromism of absorption and fluorescence spectra: a comparison with the electric-field-induced second-harmonic generation technique," Spectrochim. Acta Part A: Mol. Biomol. Spectrosc., vol. 57, no. 7, pp. 1417-1426, 2001.

[162] P. Günter, Nonlinear Optical Effects and Materials, Springer, 2012, p. 550.

[163] M. Rana, A. Chatterjee, and P. Chowdhury, "Investigation of nonlinear optical properties of organic based di amine substituted tetraphenylethylene," AIP Conf. Proc., vol. 2009, no. 1, 2018, Art no. 020055. 2018. https://doi.org/10.1063/1. 5052124.

[164] Y. Zhang, M. Jiang, J. Li, B. Hu, B. Z. Tang, and K. S. Wong, "Z-scan study of nonlinear optical standards and D-A fluorophores considering fifth-order optical nonlinearities," J. Photon. Energy, vol. 8, no. 3, 2018, https://doi.org/10.1117/1. jpe.8.032202.

[165] A. M. Nawar and I. S. Yahia, "Fabrication and characterization of anthracene thin films for wide-scale organic optoelectronic applications based on linear/ nonlinear analyzed optical dispersion parameters," Opt. Mater., vol. 70, pp. 1-10, 2017.

[166] A. Abdolmaleki, M. Dadsetani, and A. Zabardasti, “Improving the first hyperpolarizability of anthracene through interaction with $\mathrm{HX}$ molecules (XF, $\mathrm{Cl}, \mathrm{Br}$ ): a theoretical study," Spectrochim. Acta Part A: Mol. Biomol. Spectrosc., vol. 196, pp. 353-365, 2018.

[167] T. M. Pritchett, "Nonlinear refractive index of an array of azimuthal anthracene rotors". Proc of SPIE vol. 5935, 2005. https://doi.org/10.1117/12.615729.

[168] M. R. Habib, H. Li, Y. Kong, et al., "Tunable photoluminescence in a van der Waals heterojunction built from a MoS2 monolayer and a PTCDA organic semiconductor," Nanoscale, vol. 10, no. 34, pp. 16107-16115, 2018.

[169] N. Wickremasinghe, X. Wang, H. Schmitzer, and H. P. Wagner, "Eliminating thermal effects in z-scan measurements of thin PTCDA films," Opt. Express, OE., vol. 22, no. 20, pp. 2395523964, 2014.

[170] C. Liu, G. Yang, Y. Si, and X. Pan, "Photophysical properties of chiral tetraphenylethylene derivatives with the fixed propellerlike conformation,"J. Phys. Chem. C, vol. 122, no. 9, pp. 50325039, 2018

[171] N. Bian, Q. Chen, X.-L. Qiu, A.-D. Qi, and B.-H. Han, “Imidazolebearing tetraphenylethylene: fluorescent probe for metal ions based on AIE feature," New J. Chem., vol. 35, no. 8, pp. 16671671, 2011.

[172] H.-T. Feng, Y.-X. Yuan, J.-B. Xiong, Y.-S. Zheng, and B. Z. Tang, "Macrocycles and cages based on tetraphenylethylene with aggregation-induced emission effect," Chem. Soc. Rev., vol. 47, no. 19, pp. 7452-7476, 2018.

[173] T. Jadhav, J. M. Choi, B. Dhokale, S. M. Mobin, J. Y. Lee, and R. Misra, "Effect of end groups on mechanochromism and electroluminescence in tetraphenylethylene substituted phenanthroimidazoles," J. Phys. Chem. C C, vol. 120, no. 33, pp. 18487-18495, 2016.

[174] T. Jadhav, B. Dhokale, Y. Patil, S. M. Mobin, and R. Misra, "Multi-stimuli responsive donor acceptor tetraphenylethylene substituted benzothiadiazoles," J. Phys. Chem. C, vol. 120, no. 42, pp. 24030-24040, 2016.

[175] D. D. La, A. Anuradha, A. K. Hundal, S. V. Bhosale, L. A. Jones, and S. V. Bhosale, "pH-Dependent self-assembly of water-soluble sulfonate-tetraphenylethylene with aggregation-induced emission," Supramol. Chem., vol. 30, no. 1, pp. 1-8, 2018.

[176] S. Peng, J. Wen, M. Hai, et al., "Synthesis and application of reversible fluorescent photochromic molecules based on tetraphenylethylene and photochromic groups," New J. Chem., vol. 43, no. 2, pp. 617-621, 2019.

[177] C. Schilling and E. Hilinski, "Dependence of the lifetime of the twisted excited singlet-state of tetraphenylethylene on solvent polarity," J. Am. Chem. Soc., vol. 110, no. 7, pp. 2296-2298, 1988.

[178] J.-B. Xiong, H.-T. Feng, J.-P. Sun, et al., "The fixed propeller-like conformation of tetraphenylethylene that reveals aggregationinduced emission effect, chiral recognition, and enhanced chiroptical property," J. Am. Chem. Soc., vol. 138, no. 36, pp. 11469-11472, 2016.

[179] X. Zhang, Z. Chi, H. Li, et al., "Synthesis and properties of novel aggregation-induced emission compounds with combined tetraphenylethylene and dicarbazolyl triphenylethylene moieties,"J. Mater. Chem., vol. 21, no. 6, pp. 1788-1796, 2011.

[180] Y. Wang, H. Yao, J. Zhou, et al., "A water-soluble, AlE-active polyelectrolyte for conventional and fluorescence lifetime imaging of mouse neuroblastoma neuro-2A cells,"J. Polym. Sci. A-Pol. Chem., vol. 56, no. 6, pp. 672-680, 2018.

[181] R. Hu, Y. Kang, and B. Z. Tang, "Recent advances in AIE polymers," Polymer J., vol. 48, no. 4, SI, pp. 359-370, 2016.

[182] Y. Shao, Z. Gan, E. Epifanovsky, et al., "Advances in molecular quantum chemistry contained in the Q-Chem 4 program package," Mol. Phys., vol. 113, no. 2, pp. 184-215, 2015.

[183] J. Kong, C. White, A. Krylov, et al., "Q-chem 2.0: a highperformance ab initio electronic structure program package," J. Comput. Chem., vol. 21, no. 16, pp. 1532-1548, 2000.

[184] Y. Shao, L. F. Molnar, Y. Jung, et al., "Advances in methods and algorithms in a modern quantum chemistry program package," Phys. Chem. Chem. Phys., vol. 8, no. 27, pp. 3172-3191, 2006.

[185] K. Yu. Suponitsky, S. Tafur, and A. E. Masunov, "Applicability of hybrid density functional theory methods to calculation of molecular hyperpolarizability," J. Chem. Phys., vol. 129, no. 4, 2008, https://doi.org/10.1063/1.2936121.

[186] S. Draguta, M. S. Fonari, A. E. Masunov, et al., "New acentric materials constructed from aminopyridines and 4-nitrophenol," Crystencomm, vol. 15, no. 23, pp. 4700-4710, 2013.

[187] I. A. Mikhailov, M. V. Bondar, K. D. Belfield, and A. E. Masunov, "Electronic properties of a new two-photon absorbing fluorene derivative: the role of Hartree-Fock exchange in the density functional theory design of improved nonlinear chromophores," J. Phys. Chem. C, vol. 113, no. 48, pp. 2071920724, 2009.

[188] K. Yu. Suponitsky, Y. Liao, and A. E. Masunov, "Electronic hyperpolarizabilities for donor-acceptor molecules with long conjugated bridges: calculations versus experiment," J. Phys. Chem. A., vol. 113, no. 41, pp. 10994-1, 2009.

[189] K. Yu. Suponitsky, A. E. Masunov, and M. Yu. Antipin, "Computational search for nonlinear optical materials: are polarization functions important in the hyperpolarizability 
predictions of molecules and aggregates?," Mendeleev Commun., vol. 19, no. 6, pp. 311-313, 2009.

[190] I. D. Yushina, V. I. Batalov, E. V. Bartashevich, A. O. Davydov, P. S. Zelenovskiy, and A. E. Masunov, "Raman spectroscopy and theoretic study of hyperpolarizability effect in diiodobutenylbis-thioquinolinium triiodide at low temperature," J. Raman Spectrosc., vol. 48, no. 11, SI, pp. 1411-1413, 2017.

[191] K. D. Belfield, M. V. Bondar, F. E. Hernandez, et al., “Two-photon absorption properties of new fluorene-based singlet oxygen photosensitizers," J. Phys. Chem. C, vol. 113, no. 11, pp. 47064711, 2009.

[192] L. De Boni, C. Toro, A. E. Masunov, and F. E. Hernandez, "Untangling the excited states of DR1 in solution: an experimental and theoretical study," J. Phys. Chem. A, vol. 112, no. 17, pp. 3886-3890, 2008.

[193] A. Masunov, S. Tretiak, J. Hong, B. Liu, and G. Bazan, "Theoretical study of the effects of solvent environment on photophysical properties and electronic structure of paracyclophane chromophores," J. Chem. Phys., vol. 122, no. 22, 2005, https://doi.org/10.1063/1.1878732.

[194] K. Yu. Suponitsky, A. E. Masunov, and M. Yu. Antipin, "Conformational dependence of the first molecular hyperpolarizability in the computational design of nonlinear optical materials for optical switching," Mendeleev Commun., vol. 18, no. 5, pp. 265-267, 2008.

[195] A. Mourot, M. A. Kienzler, M. R. Banghart, et al., "Tuning photochromic ion channel blockers," ACS Chem. Neurosci., vol. 2, no. 9, pp. 536-543, 2011.

[196] L. Hui, C. Huang, X. Zhao, X. Xie, L. Xu, and T. Li, “Molecular design and Langmuir-Blodgett-film studies on a series of new nonlinear-optical rare-earth complexes," Langmuir, vol. 10, no. 10, pp. 3794-3796, 1994.

[197] H. Li, C. Huang, Y. Zhao, et al., "Lanmuir-Blodgett-film and 2nd harmonic generation of a series of new nonlinear-optical rareearth complexes," Solid State Commun., vol. 94, no. 9, pp. 731733, 1995.

[198] X. Zhao, X. Xie, X. Xia, et al., "Effect of gas environment on 2nd harmonic generation of Langmuir-Blodgett-films," Thin Solid Films, vol. 263, no. 1, pp. 13-15, 1995.

[199] H. Li, C. Huang, X. Zhao, X. Xie, L. Xu, and T. Li, “Molecular design and Langmuir-blodgett film studies on a series of new nonlinear optical rare earth complexes," Langmuir, vol. 10, no. 10, pp. 3794-3796, 1994.

[200] H. Li, C. Huang, Y. Zhao, et al., "Langmuir-Blodgett film and second harmonic generation of a series of new nonlinear optical rare earth complexes," Solid State Commun., vol. 94, no. 9, pp. 731-733, 1995.

[201] X. Zhao, X. Xie, X. Xia, et al., "Effect of gas environment on second harmonic generation of Langmuir-Blodgett films," Thin Solid Films, vol. 263, no. 1, pp. 13-15, 1995.

[202] D. Marcuse, "Mode conversion caused by surface imperfections of a dielectric slab waveguide," Bell System Tech. J., vol. 48, no. 10, p. 3187+, 1969.

[203] A. Chutinan and S. Noda, "Waveguides and waveguide bends in two-dimensional photonic crystal slabs," Phys. Rev. B, vol. 62, no. 7, pp. 4488-4492, 2000.

[204] D. Mori and T. Baba, "Wideband and low dispersion slow light by chirped photonic crystal coupled waveguide," Opt. Express, vol. 13, no. 23, pp. 9398-9408, 2005.
[205] M. Notomi, A. Shinya, K. Yamada, J. Takahashi, C. Takahashi, and I. Yokohama, "Structural tuning of guiding modes of linedefect waveguides of silicon-on-insulator photonic crystal slabs," IEEE J. Quantum Electron., vol. 38, no. 7, pp. 736-742, 2002.

[206] V. Braginsky, M. Gorodetsky, and V. Ilchenko, "Quality-factor and nonlinear properties of optical whispering-gallery modes," Phys. Lett. A., vol. 137, nos 7-8, pp. 393-397, 1989.

[207] V. Ilchenko, A. Savchenkov, A. Matsko, and L. Maleki, "Nonlinear optics and crystalline whispering gallery mode cavities," Phys. Rev. Lett., vol. 92, no. 4, 2004, https://doi.org/ 10.1103/physrevlett.92.043903.

[208] I. S. Grudinin, A. B. Matsko, and L. Maleki, "Brillouin lasing with a CaF2 whispering gallery mode resonator," Phys. Rev. Lett., vol. 102, no. 4, 2009, Art no. 043902.

[209] S. Soltani, V. M. Diep, R. Zeto, and A. M. Armani, "Stimulated anti-Stokes Raman emission generated by gold nanorod coated optical resonators," ACS Photon., vol. 5, no. 9, pp. 3550-3556, 2018.

[210] H. Choi, D. Chen, F. Du, R. Zeto, and A. Armani, "Low threshold anti-Stokes Raman laser on-chip,” Photon. Res., vol. 7, no. 8, pp. 926-932, 2019.

[211] X. Xue, F. Leo, Y. Xuan, et al., "Second-harmonic-assisted fourwave mixing in chip-based microresonator frequency comb generation," Light: Sci. Appl., vol. 6, no. 4, 2017, Art no. e16253.

[212] P. Del'Haye, T. Herr, E. Gavartin, M. L. Gorodetsky, R. Holzwarth, and T. J. Kippenberg, "Octave spanning tunable frequency comb from a microresonator," Phys. Rev. Lett., vol. 107, no. 6, 2011, https://doi.org/10.1103/PhysRevLett.107.063901.

[213] C. Wang, M. Zhang, M. Yu, R. Zhu, H. Hu, and M. Loncar, "Monolithic lithium niobate photonic circuits for Kerr frequency comb generation and modulation," Nat. Commun., vol. 10, 2019, https://doi.org/10.1038/s41467019-08969-6.

[214] C. Xiong, W. Pernice, K. K. Ryu, et al., "Integrated GaN photonic circuits on silicon (100) for second harmonic generation," Opt. Express, vol. 19, no. 11, pp. 10462-10470, 2011.

[215] I. Roland, M. Gromovyi, Y. Zeng, et al., "Phase-matched second harmonic generation with on-chip GaN-on-Si microdisks," Sci. Rep., vol. 6, 2016, https://doi.org/10.1038/srep34191.

[216] X. Liu, C. Sun, B. Xiong, et al., "Aluminum nitride-on-sapphire platform for integrated high-Q microresonators," Opt. Express, vol. 25, no. 2, pp. 587-594, 2017.

[217] M.-C. Tien, J. F. Bauters, M. J. R. Heck, D. T. Spencer, D. J. Blumenthal, and J. E. Bowers, "Ultra-high quality factor planar $\mathrm{Si}_{3} \mathrm{~N}_{4}$ ring resonators on Si substrates," Opt. Express, vol. 19, no. 14, pp. 13551-13556, 2011.

[218] Y. Sun, W. Shin, D. A. Laleyan, et al., "Ultrahigh Q microring resonators using a single-crystal aluminum-nitride-on-sapphire platform," Opt. Lett., vol. 44, no. 23, pp. 5679-5682, 2019.

[219] Y. Sun, D. Laleyan, E. Reid, et al., High-Q Resonators on Single Crystal Aluminum Nitride Grown by Molecular Beam Epitaxy, 2019.

[220] T.-J. Lu, M. Fanto, H. Choi, et al., "Aluminum nitride integrated photonics platform for the ultraviolet to visible spectrum," Opt. Express, vol. 26, no. 9, pp. 11147-11160, 2018.

[221] X. Liu, A. W. Bruch, J. Lu, et al., "Beyond 100 THz-spanning ultraviolet frequency combs in a non-centrosymmetric crystalline waveguide," Nat. Commun., vol. 10, p. 2971, 2019. 
[222] M. Soltani, R. Soref, T. Palacios, and D. Englund, "AlGaN/AIN integrated photonics platform for the ultraviolet and visible spectral range," Opt. Express, vol. 24, no. 22, pp. 25415-25423, 2016.

[223] J. B. Surya, X. Guo, C.-L. Zou, and H. X. Tang, "Efficient thirdharmonic generation in composite aluminum nitride/silicon nitride microrings," Optica, vol. 5, no. 2, pp. 103-108, 2018.

[224] D. Y. Oh, K. Y. Yang, C. Fredrick, G. Ycas, S. A. Diddams, K. J. Vahala "Coherent ultra-violet to near-infrared generation in silica ridge waveguides". Nat. Commun., vol. 8, 2017, doi: https://doi.org/10.1038/ncomms13922.

[225] H. Chen, J. Zhou, D. Li, et al., "On-chip directional octavespanning supercontinuum generation from high order mode in near ultraviolet to infrared spectrum using AIN waveguides," Work. Pap.

[226] J. Dudley and S. Coen, "Numerical simulations and coherence properties of supercontinuum generation in photonic crystal and tapered optical fibers," IEEEJ. Sel. Top. Quantum Electron., vol. 8, no. 3, pp. 651-659, 2002.

[227] S. D. S. Rao, R. D. Engelsholm, I. B. Gonzalo, et al., "Ultra-lownoise supercontinuum generation with a flat near-zero normal dispersion fiber," Opt. Lett., vol. 44, no. 9, pp. 2216-2219, 2019 , https://doi.org/10.1364/OL.44.002216.

[228] I. B.Gonzalo, R. D. Engelsholm, M. P. Sorensen, O. Bang "Polarization noise places severe constraints on coherence of all-normal dispersion femtosecond supercontinuum generation,". Sci. Rep., vol. 8 2018, https://doi.org/10.1038/ s41598-018-24691-7.

[229] T. Ling, S.-L. Chen, and L. J. Guo, “Fabrication and characterization of high $\mathrm{Q}$ polymer micro-ring resonator and its application as a sensitive ultrasonic detector," Opt. Express, vol. 19, no. 2, pp. 861-869, 2011.

[230] H. S. Choi and A. M. Armani, "Thermal nonlinear effects in hybrid optical microresonators," Appl. Phys. Lett., vol. 97, no. 22, p. 223306, 2010.

[231] H. S. Choi, X. Zhang, and A. M. Armani, "Hybrid silica-polymer ultra-high-Q microresonators,” Opt. Lett., vol. 35, no. 4, p. 459, 2010.

[232] X. Shen, R. C. Beltran, V. M. Diep, S. Soltani, and A. M. Armani, "Low-threshold parametric oscillation in organically modified microcavities," Sci. Adv., vol. 4, no. 1, 2018, Art no.eaao4507.

[233] B. W. Biggs, H. K. Hunt, and A. M. Armani, "Selective patterning of Si-based biosensor surfaces using isotropic silicon etchants," J. Colloid. Interface. Sci., vol. 369, no. 1, pp. 477481, 2012.

[234] H. K. Hunt, C. Soteropulos, and A. M. Armani, "Bioconjugation strategies for microtoroidal optical resonators," Sensors, vol. 10, no. 10, pp. 9317-9336, 2010.

[235] H. S. Choi, D. Neiroukh, H. K. Hunt, and A. M. Armani, "Thermooptic coefficient of polyisobutylene ultrathin films measured with integrated photonic devices," Langmuir, vol. 28, no. 1 , pp. 849-854, 2012.

[236] C. T. Herman, G. K. Potts, M. C. Michael, N. V. Tolan, and R. C. Bailey, "Probing dynamic cell-substrate interactions using photochemically generated surface-immobilized gradients: application to selectin-mediated leukocyte rolling," Int. Bio. (Cam), vol. 3, no. 7, pp. 779-791, 2011

[237] J.-Y. Byeon, F. T. Limpoco, and R. C. Bailey, "Efficient bioconjugation of protein capture agents to biosensor surfaces using aniline-catalyzed hydrazone ligation," Langmuir, vol. 26, no. 19, pp. 15430-15435, 2010.

[238] A. L. Washburn, L. C. Gunn, and R. C. Bailey, "Label-free quantitation of a cancer biomarker in complex media using silicon photonic microring resonators," Anal. Chem., vol. 81, no. 22, pp. 9499-9506, 2009.

[239] J.-H. Yoo, S. Rafique, A. Lange, H. Zhao, and S. Elhadj, “Lifetime laser damage performance of beta-Ga203 for high power applications," APL Mater., vol. 6, no. 3, 2018, https://doi.org/ 10.1063/1.5021603.

[240] J. Zhou, H. Chen, H. Fu, et al., "Demonstration of low loss betaGa203 optical waveguides in the UV-NIR spectra," Appl. Phys. Lett., vol. 115, no. 25, 2019, https://doi.org/10.1063/1.5133845.

[241] J. Zhang, B. Li, C. Xia, et al., "Growth and spectral characterization of beta-Ga203 single crystals," J. Phys. Chem. Solids, vol. 67, no. 12, pp. 2448-2451, 2006.

[242] H. Reis, M. G. Papadopoulos, P. Calaminici, K. Jug, and A. M. Köster, "Calculation of macroscopic linear and nonlinear optical susceptibilities for the naphthalene, anthracene and meta-nitroaniline crystals," Chem. Phys., vol. 261, no. 3, pp. 359-371, 2000

[243] P. S. Patil, S. R. Maidur, J. R. Jahagirdar, T. S. Chia, C. K. Quah, and M. Shkir, "Crystal structure, spectroscopic analyses, linear and third-order nonlinear optical properties of anthracenebased chalcone derivative for visible laser protection," Appl. Phys. B., vol. 125, no. 9, p. 163, 2019.

[244] R. Gostl and R. P. Sijbesma, "pi-extended anthracenes as sensitive probes for mechanical stress," Chem. Sci., vol. 7, no. 1, pp. 370-375, 2016.

[245] M. Liu, H. S. Quah, S. Wen, et al., "Nonlinear optical properties of a one-dimensional coordination polymer," J. Mater. Chem. C., vol. 5, no. 11, pp. 2936-2941, 2017.

[246] N. T. Binh, B.-P. Zhang, C.-Y. Liu, et al., "Structural and optical properties of $\mathrm{ZnO}$ epitaxial films grown on Al203 (11220) substrates by metalorganic chemical vapor deposition," Jpn. J. Appl. Phys., vol. 43, no. 7R, p. 4110, 2004.

[247] J. He, A. Kovach, D. Chen, P. J. G. Saris, R. Yu, and A. M. Armani, "All-optical reversible controls of integrated photonics by selfassembled azobenzene,"arXiv:2001.01114 [physics], 2020.

[248] A. Kovach, J. He, P. Saris, D. Chen, and A. M. Armani, "Optically tunable microresonator using an azobenzene monolayer," AIP Adv., vol. 10, no. 4, 2020, Art no. 045117.

[249] S. Molesky, Z. Lin, A. Y. Piggott, W. Jin, J. Vucković, and A. W. Rodriguez, "Inverse design in nanophotonics," Nat. Photon., vol. 12, no. 11, pp. 659-670, 2018.

[250] B. Shen, P. Wang, R. Polson, and R. Menon, "An integratednanophotonics polarization beamsplitter with $2.4 \times 2.4 \mu \mathrm{m}$ 2 footprint," Nat. Photon., vol. 9, no. 6, pp. 378-382, 2015.

[251] J. C. C. Mak, C. Sideris, J. Jeong, A. Hajimiri, and J. K. S. Poon, "Binary particle swarm optimized $2 \times 2$ power splitters in a standard foundry silicon photonic platform," Opt. Lett., vol. 41, no. 16, pp. 3868-3871, 2016.

[252] M. Giles and N. Pierce, "An introduction to the adjoint approach to design," Flow Turbul. Combust., vol. 65, nos 3-4, pp. 393-415, 2000.

[253] C. M. Lalau-Keraly, S. Bhargava, O. D. Miller, and E. Yablonovitch, "Adjoint shape optimization applied to electromagnetic design," Opt. Express, vol. 21, no. 18, pp. 21693-21701, 2013. 
[254] T. P. Xiao, O. S. Cifci, S. Bhargava, et al., "Diffractive spectralsplitting optical element designed by adjoint-based electromagnetic optimization and fabricated by femtosecond 3D direct laser writing," ACS Photon., vol. 3, no. 5, pp. 886894, 2016.

[255] L. Su, A. Y. Piggott, N. V. Sapra, J. Petykiewicz, and J. Vuckovic, "Inverse design and demonstration of a compact on-chip narrowband three-channel wavelength demultiplexer," ACS Photon., vol. 5, no. 2, pp. 301-305, 2018.

[256] Y. Elesin, B. S. Lazarov, J. S. Jensen, and O. Sigmund, “Design of robust and efficient photonic switches using topology optimization," Photon. Nanostruc. Fundam. Appl., vol. 10, no. 1, pp. 153-165, 2012.

[257] J. Lu, S. Boyd, and J. Vuckovic, "Inverse design of a threedimensional nanophotonic resonator," Opt. Express, vol. 19, no. 11, pp. 10563-10570, 2011.

[258] A. Y. Piggott, J. Lu, T. M. Babinec, K. G. Lagoudakis, J. Petykiewicz, and J. Vuckovic, "Inverse design and implementation of a wavelength demultiplexing grating coupler," Sci. Rep., vol. 4, 2014, https://doi.org/10.1038/srep07210.

[259] A. Y. Piggott, J. Petykiewicz, L. Su, and J. Vuckovic, "Fabricationconstrained nanophotonic inverse design,” Sci. Rep., vol. 7, 2017, https://doi.org/10.1038/s41598-017-01939-2.

[260] A. Oskooi, A. Mutapcic, S. Noda, J. D. Joannopoulos, S. P. Boyd, and S. G. Johnson, "Robust optimization of adiabatic tapers for coupling to slow-light photonic-crystal waveguides," opt. Express, vol. 20, no. 19, pp. 21558-21575, 2012.
[261] C. Sideris, E. Garza, and O. P. Bruno, "Ultrafast simulation and optimization of nanophotonic devices with integral equation methods," ACS Photon., vol. 6, no. 12, pp. 3233-3240, 2019.

[262] C. Dory, D. Vercruysse, K. Y. Yang, et al., "Inverse-designed diamond photonics," Nat. Commun., vol. 10, 2019, https://doi. org/10.1038/s41467-019-11343-1.

[263] T. W. Hughes, M. Minkov, I. A. D. Williamson, and S. Fan, "Adjoint method and inverse design for nonlinear nanophotonic devices," ACS Photon., vol. 5, no. 12, pp. 47814787, 2018.

[264] R. E. Christiansen, F. Wang, and O. Sigmund, "Topological insulators by topology optimization," Phys. Rev. Lett., vol. 122, no. 23 , 2019, https://doi.org/10.1103/physrevlett.122. 234502.

[265] A. Taflove, and S. C. Hagness, Computational Electrodynamics: The Finite-difference Time-domain Method, Arteh House, 2005.

[266] Y. Shi, W. Shin, and S. Fan, "Multi-frequency finite-difference frequency-domain algorithm for active nanophotonic device simulations," Optica, vol. 3, no. 11, pp. 1256-1259, 2016.

[267] K. Y. Yang, J. Skarda, M. Cotrufo, et al., "Inverse-designed photonic circuits for fully passive, bias-free Kerr-based nonreciprocal transmission and routing," arXiv:1905.04818 [physics], 2019.

[268] J. Wang, Y. Shi, T. Hughes, Z. Zhao, and S. Fan, "Adjoint-based optimization of active nanophotonic devices," Opt. Express, vol. 26, no. 3, pp. 3236-3248, 2018. 